Revista Mexicana de Economía y Finanzas Nueva Época

Volumen 14 Número 2, Abril-Junio 2019, pp. 279-307

DOI: https://doi.org/10.21919/remef.v14i2.310

\title{
Análisis comparativo de las metodologías de estimación semiparamétricas y vía cópulas del Valor en Riesgo (VaR) en el mercado accionario colombiano
}

\author{
Miguel Antonio Alba Suárez ${ }^{1}$ \\ Universidad Santo Tomás, Colombia \\ Wilmer Pineda-Ríos ${ }^{2}$ \\ Universidad Santo Tomás, Colombia \\ Javier Deaza Chaves ${ }^{3}$ \\ Universidad Santo Tomás, Colombia \\ (Primera recepción: 21/junio/2018, última recepción: 30/enero/2019, \\ aceptado: 22/febrero/2019)
}

\section{Resumen}

Este articulo de investigación ilustra distintos tipos de metodologías estadísticas con el objetivo de realizar una estimación adecuada para el valor en riesgo (VaR), implementando el uso de metodos semiparamétricos y una clase flexible de cópulas nombradas como (VineCopulas) encontrando que en las tecnicas de estimación al incluir el manejo de los patrones complejos de dependencia no lineal en el modelado de los activos financieros, se logra explicar la volatilidad y los movimientos dinámicos del mercado. La flexibilidad de los modelos presentados con el uso de Cópulas y metodologías semiparamétricas como la quasiverosimilitud (QMLE) y teoría de valor extremo (EVT) permitió la adecuada estimación del VaR en el mercado de renta variable Colombiano.

Clasificación JEL: C02, C06, C11, C14, G32

Palabras clave: Mercado accionario, EVT, QMLE, Semiparamétrico Valor en Riesgo, Vine Cópulas

\section{Comparative Analysis of the Semi-parametric Estimation Methodologies and Copula Estimation in Value at Risk (VaR) in the Colombian Stock Market}

\section{Abstract}

This research article illustrates different types of statistical methodologies with the objective of making an adequate estimate for value at risk (VaR), implementing the use of semi-parametric methods and a flexible class of copulas named VineCopulas. It was found that it is possible to explain volatility and dynamic market movements in estimation techniques by including the management of complex patterns of non-linear dependence in the modeling of financial assets. The flexibility of the models presented with the use of copulas and semi-parametric methodologies, such as quasi-maximum likelihood estimate (QMLE) and extreme value theory (EVT), allowed the adequate estimation of VaR in

\footnotetext{
${ }^{1} \mathrm{PhD}$ (c) en Administración, MSc en Ciencias Económicas, Economista, Docente Investigador de la Facultad de Negocios Internacionales Universidad Santo Tomás-Bogotá. Teléfono 3168775728. e-mail: miguelalba@usantotomas.edu.co.

${ }^{2} \mathrm{PhD}$ (c) en Estadistica, MSc en Matemáticas, Matemático, Coordinador del Centro de Investigaciones de la Facultad de Estadística Universidad Santo Tomás-Bogotá. Teléfono 3005985899. e-mail: wilmerpineda@usantotomas.edu.co.

${ }^{3}$ MSc en Economía, Economista, Docente Investigador de la Facultad de la Facultad de Negocios Inyternacionales Universidad Santo Tomás-Bogotá. Teléfono 3103025545. e-mail: javierdeaza@usantotomas.edu.co.
} 
REMEF (The Mexican Journal of Economics and Finance) Análisis comparativo de las metodologías de estimación semiparamétricas $280 \quad$ y vía cópulas del Valor en Riesgo (VaR) en el mercado accionario colombiano

\section{Abstract}

the Colombian equity market.

JEL Classification: C02, C06, C11, C14, G32

Keywords: Equity Market, EVT, QMLE, Semi-parametric Value at Risk, VineCopulas

\section{Introducción}

En los mercados financieros, los inversionistas se encuentran expuestos a un sinnúmero de riesgos entre los que se encuentran el riesgo de crédito, riesgo operacional, riesgo de liquidez y riesgo de mercado entre otros. Si bien, estos riesgos son objeto de estudio por parte del mercado, se hace necesario conocer las diferentes metodologías de estimación del valor en riesgo en distintos marcos de tiempo; para tal efecto, se presenta un análisis comparativo de las metodologías de estimación semiparamétricas y vine cópulas del valor en riesgo (VaR) del mercado de renta variable en Colombia para el período 2015-2017.

Las metodologías por desarrollar son no convencionales debido a que, tradicionalmente el sistema financiero ha venido utilizando en la estimación del VaR (Valor en Riesgo): la simulación histórica y montecarlo entre otras, las cuales no toman en cuenta la dependencia no lineal entre los datos causando varios problemas en la gestión de riesgos.

Consecuencia de lo anterior, se hace una breve revisión teórica a nivel nacional como internacional de los principales expositores que han abordado el estudio del valor en riesgo bajo las diferentes metodologías. Posteriormente se hace un análisis teórico de las metodologías semiparamétricas y vine cópulas para finalizar con un trabajo aplicado al mercado de renta variable colombiano utilizando las metodologías no convencionales de estimación del VaR.

\section{Literatura Nacional e Internacional}

En Colombia, la estimación del valor en riesgo en activos financieros ha sido impuesta a las entidades financieras, quienes han venido asumiendo está medición a través de la implementación de la circular básica contable y financiera de la Superintendencia Financiera No XXI (Supefinanciera, 2007).

En lo que atañe a la medición del VaR, de acuerdo con, las directrices de la Superfinanciera, ha sido sujeta a críticas por parte de las entidades financieras, quienes ven este procedimiento, un poco alejado de la realidad en la medida en que los eventos de los últimos años se ha caracterizado por altos niveles de volatilidad, que han generado pérdida de credibilidad en su estimación; por lo tanto, las estimaciones que han realizado o vienen realizando las entidades distan de lo que está sucediendo en la realidad (Caicedo \& Enrique, 2014).

De conformidad con lo anterior, se hace necesario explicitar otro tipo de metodologías que se alejen de lo convencional y que de alguna manera expongan mejor la realidad del mercado de renta variable colombiano.

\subsection{Literatura Internacional}

Los autores que han aportado en la construcción de metodologías no convencionales en la estimación del valor en riesgo $(\mathrm{VaR})$ son los siguientes:

1) Thomas Breuer, quien facilitó la conceptualización y las metodologías matemáticas para la utilización de la teoría del valor extremo (EVT), valor extremo condicional (EVC) y valores extremos en modelos con volatilidad (Feria, J \& Oliver, M, 2006).

2) Mathias Hofmann y Claudia Czado, proponen el uso de VineCopulas utilizando estimaciones bayesianas para modelos de tipo GARCH multivariado en la estimación del valor en riesgo (Hofmann, M \& Czado, C, 2011). 
3) Jostein Larsen Benterud, Magnus Solli Haukaas y Paul Ingebrigt Huse, proponen el uso del modelado de vine-cópulas para describir el comportamiento de modelos de riesgo comparando las estimaciones en el valor en riesgo con las metodologías clásicas (Larsen, J; Solli M \& Ingebrigt P, 2013).

\subsection{Literatura Nacional}

En la literatura local los autores que han estudiado la estimación del riesgo de mercado son las siguientes:

1) Luis Fernando Melo y Oscar Becerra Camargo proponen una estimación del VaR para la tasa interbancaria de Colombia utilizando metodologías ARMA-GARCH-EVT en donde identifican las técnicas con mejor y peor desempeño en la estimación (Melo V, Luis Becerra C, Oscar, 2005).

2) Daniel Mariño Ustacara y Luis Fernando Melo proponen la utilización de métodos semiparamétricos mediante el uso de la regresión cuantílica lineal y no lineal comparándolas con las técnicas tradicionales para la estimación del VaR en activos financieros (Mariño, D Melo, L, 2016).

3) Pamela Cardozo propone la estimación del valor en riesgo en activos financieros utilizando la metodología de la teoría del valor extremo en la estimación del VaR comparándola con el método delta-normal. (Cardozo, 2004).

A continuación, se exponen los métodos no clásicos de estimación del valor en riesgo a nivel teórico:

\section{Metodologías semiparamétricas y Vine cópulas}

El cálculo del VaR frecuentemente necesita del modelamiento de la volatilidad de los retornos de los activos financieros por medio de metodologías que asumen distribuciones sobre el logaritmo de los retornos de los precios de los activos (metodologías perimétricas), y aquellas metodologías que no asumen ningún tipo de comportamiento para los retornos. Las metodologías semi-paramétricas permiten la flexibilidad para adaptar las técnicas de estimación a los movimientos dinámicos del mercado (Melo V, Luis Becerra C, Oscar, 2005)

\subsection{Estimación del VaR usando la Teoría de Valor Extremo (EVT)}

Esta metodología, parte de asumir dependencia en el cálculo del VaR, tomando en cuenta eventos extremos como son la dependencia en las colas de distribución. Este método es uno de los más acertados para estimar el VaR, al contar con soluciones para generar decai-

mientos en la heterocedasticidad, y correlación serial dada por modelos de tipo GARCH multivariado (Mariño, D Melo, L, 2016).

La teoría del valor extremo nació como uno de las disciplinas más grandes en la estadística aplicada en los últimos 50 años, por su capacidad para cuantificar la conducta de procesos a niveles grandes o pequeños. (Cardozo, 2004)

Los indicios de la estimación del valor en riesgo a través del EVT buscan de algún modo, cuantificar el comportamiento de las pérdidas a través de la probabilidad mediante el análisis del comportamiento de las colas de la distribución de los retornos dados por un portafolio de inversión. Este método semiparamétrico, proporciona medidas más fuertes en cuanto a las colas de la distribución de los retornos obtenidos mediante comportamientos extremos en los datos, que no solucionan las medidas paramétricas y no paramétricas.

La EVT ofrece un buen modelado de los datos utilizando el comportamiento extremo de las colas de las distribuciones de pérdidas asociadas a las observaciones a través de estimaciones con asociaciones paramétricas que permiten encontrar la cola de la distribución. 
REMEF (The Mexican Journal of Economics and Finance) Análisis comparativo de las metodologías de estimación semiparamétricas

Según (Fernandez, 2003) el comportamiento actual de la EVT está basado en el identificar momentos estadísticos de los retornos de un portafolio de inversión, así como el cálculo de la distribución de los retornos financieros; sin embargo, para este trabajo se centrará en el cálculo de aplicaciones multivariadas en la estimación del valor en riesgo.

La teoría del valor extremo parte de los siguientes supuestos y condiciones:

Sea $F_{X}(x)$ la función de distribución acumulada desconocida para los retornos negativos del portafolio de inversión seleccionado. Se le dice evento extremo a las pérdidas generadas por la cola derecha de la distribución de pérdidas, y $\Phi_{n}$ al máximo de los retornos negativos conocidos como riesgos; este último conocido como el peor caso de pérdidas en la muestra aleatoria de $\mathrm{n}$ riesgos

La distribución de $\Phi_{n}$ según (Fernandez, 2003) se escribe de la siguiente manera ${ }^{4}$ :

$$
P\left(\Phi_{n}<x\right)=P\left(X_{1}<x_{1}, X_{2}<x_{2}, \ldots, X_{n}<x_{n}\right)=\prod_{i=1}^{n} F_{\Phi_{n}}(X)=F^{n}(x)
$$

Para el cálculo del valor en riesgo, a través de esta metodología se deben tener en cuenta los siguientes supuestos sobre la teoría del valor extremo:

1) Para límites muy altos la cola de la distribución de los datos converge a la distribución generalizada de Pareto $^{5}$.

$$
G_{\rho}, \beta=\left\{\begin{array}{c}
1-\left(1+\frac{\rho x}{\beta}\right)^{-\frac{1}{\rho}}, \rho \neq 0 \\
1-e^{-\frac{x}{\beta}}, \rho=0
\end{array}\right.
$$

2) Se posee una distribución de excesos o extremos por encima del umbral $\theta$ generando la siguiente probabilidad condicional:

$$
F(\delta)=P(X-\theta \leq \delta \mid X>\theta)=\frac{F(\delta-\theta)-F(\theta)}{1-F(\theta)}
$$

Lo que permite que para valores grandes de $\theta$ exista una función $\beta(\theta)$ que garantice el limite

$$
\lim \theta \rightarrow X_{0} \sup _{0 \leq \delta} X_{0}-\theta\left|F(\delta)-G_{\rho}, \beta(\theta)\right|=0
$$

Donde $X_{0}$ corresponde a la pérdida máxima en la distribución.

Lo anterior implica, que a largas distribuciones el dominio máximo de atracción sea de tipo: normal, log normal, chi-cuadrado, t, gamma, f, etc.

Asumiendo los anteriores aspectos, es necesario el cálculo de las colas de la distribución de pérdida, teniendo en cuenta como se mencionó antes, que a cantidades de umbral $\theta$ la distribución de excesos $F(\delta)$ converge a la distribución Pareto generalizada $G_{\rho}, \beta$.

Siguiendo lo anterior, se puede encontrar la aproximación para la distribución $F_{X}(x)$ como:

$$
F_{X}(x)=(1-F(\theta)) G_{\rho}, \beta(\theta)+F(\theta)
$$

Donde la función del umbral $\theta$ se puede estimar de manera no paramétrica usando la distribución empírica 1-r/n, donde $\mathrm{r}$ representa las realizaciones hechas por encima del umbral $\theta$.

\footnotetext{
${ }^{4}$ Usando el teorema de Fisher-Tippet dado un límite superior para F se encuentra un valor máximo estandarizado $\mathrm{Z}$ generando los parámetros de forma y escala $\sigma, \mu$. Lo explicitado garantiza que el estadístico $\mathrm{Z}$ converja a una distribución no degenerativa; a través de lo anterior, surge un acercamiento mejor para modelar los valores extremos propuestos por la distribución utilizando los limites extremos de la distribución lo que permite el cálculo del VaR de manera más sencilla

${ }^{5} \beta \geq 0, \rho \geq 0, x \in[0, \infty) y \rho<0, x \in\left[0, \frac{-\beta}{\rho}\right)$
} 
La estimación de la distribución se escribe de la siguiente manera:

$$
\widehat{F_{X}(x)}=1-\frac{r}{n}\left(1+\hat{\rho} \frac{(x-\theta)}{\hat{\beta}}\right)
$$

(Mariño, D Melo, L, 2016) indica que los parámetros para encontrar esta distribución se estiman vía máxima verosimilitud.

En lo que concierne a la estimación del VaR después de tener la distribución de los retornos negativos se selecciona un nivel de confianza entre $0<\operatorname{conf}<1$, y se calcula la siguiente fórmula (Hao Li, Xiao Fan, Yu Li, Yue Zhou, Ze Jin Zhao Liu, 2014)

$$
\widehat{\operatorname{VaR}_{t+1}^{\text {con } f}}=u+\frac{\hat{\beta}}{\hat{\rho}}\left(\left(\frac{n}{r}(1-\operatorname{con} f)\right)^{-\rho}-1\right)
$$

\subsection{Estimación del VaR usando Cuasi-verosimilitud}

La volatilidad es el comportamiento principal en el modelado de activos financieros; se puede observar en la práctica usualmente que ésta se aglomera y está autocorrelacionada a lo largo del tiempo. Los modelos GARCH son la mejor aproximación para explicar el comportamiento de explosión y calma en los activos, convirtiéndose en una herramienta muy útil en el manejo del riesgo financiero.

La estimación del VaR con supuestos normales, evidencian en la aplicación, una presencia amplia de sesgo y curtosis, lo que resulta en poca o mucha cobertura de las estimaciones calculadas. Por lo que asumir otro tipo de distribuciones y realizar modelos para el manejo de riesgo, resulta en muchas ocasiones un factor óptimo en la estimación oportuna del VaR (Embrechts, 2016).

En la práctica es bastante común utilizar modelos auto-regresivos de heterocedasticidad condicional (ARCH) y generalizados (GARCH) ya que estos, capturan los movimientos no constantes de la varianza a través del tiempo, luchando contra los constantes cambios en la volatilidad. En la práctica los modelos más utilizados de esta clase son los ARCH (1), GARCH $(1,1)$ y $\operatorname{EGARCH}(1,1)$; en el caso de este documento se estimará el VaR por medio del modelo $\operatorname{GARCH}(1,1)$

Sea $\phi_{t}=\ln \left(\frac{P_{t}}{P_{t-1}}\right)$ los continuos cambios en los retornos desde el período empezado en t-1 hasta t, y $P_{t}$ como el precio de los activos financieros al tiempo t; la desagregación de $\phi_{t}$ se puede escribir de la siguiente manera:

$$
\phi_{t}=E\left(\phi_{t} \mid \rho_{t-1}\right)+\varepsilon_{t}
$$

Donde $\rho_{t-1}$ corresponde a la información en el tiempo propuesto menos una unidad y $\varepsilon_{t}$ los errores de dicha desagregación considerados como valores no predictivos; el valor esperado para el $i$-simo proceso de auto-regresión se escribe como:

$$
E\left(\phi_{t} \mid \rho_{t-1}\right)=a_{0}+\sum_{j=1}^{i} a_{j} \phi_{t-j}
$$

Los valores no predictivos se pueden escribir como procesos de tipo ARCH $\varepsilon_{t}=m_{t} \sigma_{t}$, donde $m_{t}$ corresponde a un vector de medias de 0 , varianza 1 y la varianza condicional de $\varepsilon_{t}$ es $\sigma_{t}$ (Embrechts, P Hoing, A, 2006).

Con lo anterior, la varianza condicional del modelo $\mathrm{ARCH}(\mathrm{q})$ se escribe como:

$$
\sigma_{t}^{2}=c_{0}+\sum_{i=1}^{q} c_{i} \varepsilon_{t-i}^{2}
$$


REMEF (The Mexican Journal of Economics and Finance) Análisis comparativo de las metodologías de estimación semiparamétricas

Donde $c_{i} \geq 0 \forall i=1, \ldots, q$ y corresponde a la reacción a los nuevos comportamientos en el mercado; a través de la anterior definición, se logra generalizar el modelo ARCH convirtiéndolo en un $\operatorname{GARCH}(\mathrm{p}, \mathrm{q})$ con la siguiente varianza condicional:

$$
\sigma_{t}^{2}=c_{0}+\sum_{i=1}^{q} c_{i} \varepsilon_{t-i}^{2}+\sum_{d=1}^{p} \beta_{d} \sigma_{t-d}^{2}
$$

Donde si $c+\beta<1$, el proceso se considerará estacionario, y a su vez $\beta_{i}$ sostiene la persistencia de la heterocedasticidad en el tiempo. Para el caso del modelo GARCH $(1,1)$ la varianza condicional se escribe de la siguiente manera:

$$
\sigma_{t+1}^{2}=c_{0}+c_{1} \varepsilon_{t}^{2}+\beta_{1} \sigma_{t}^{2}
$$

El modelo GARCH(p,q), según (Lei, Fan J Xiu D, 2014) propone una solución bastante buena frente a problemas con la volatilidad, y las colas pesadas de la distribución de los retornos, por lo que genera estimaciones confiables al incluir este factor difícil de modelar y pronosticar.

Por otro lado, este modelo carece de eficiencia por las limitaciones que posee, en cuanto a la varianza dependiente de la magnitud y no de los $\varepsilon_{t}$; lo que no es evidente en el comportamiento de los precios donde se encuentra un factor de apalancamiento en los cuales, los activos presentan correlaciones negativas entre ellos, y en repercusión logran cambios en la volatilidad (Fan J Gu J, 2003).

En el caso, de la estimación de los parámetros de los modelos GARCH (p,q) es frecuente usar la estimación por máxima verosimilitud (MLE), asumiendo normalidad en la distribución de los errores $\varepsilon_{i t}$ del modelo ARMA previamente realizado antes de modelar la volatilidad. Por otro lado, se pueden asumir distribuciones para los errores como la t de student centrada o no, distribución generalizada del error, inversa gaussiana o la distribución de Johnson's; sin embargo, estos métodos carecen de consistencia en las estimaciones de los parámetros. En este caso la estimación por máxima cuasi-verosimilitud (QMLE) puede ser consistente y asintoticamente normal bajo condiciones que respectan al cuarto momento del proceso ARCH. La robustez en las estimaciones siempre va acompañada de eficiencia en los estimadores, por lo que la estimación por verosimilitud gaussiana asintótica en las innovaciones de los errores aumenta de varianza significativamente en las estimaciones de los parámetros por lo que el estimador se vuelve ineficiente por MLE. Sin embargo, aplicando algunas técnicas semi paramétricas por simulación de montecarlo es posible ganar eficiencia en los estimadores por medio de QMLE gaussianos (Fernandez, 2003).

En general, la técnica de estimación frecuentemente utilizada en los modelos GARCH por medio de QMLE es la Cuasi-verosimilitud Gaussiana (GQMLE) la cual garantiza la mejora en eficiencia de las estimaciones (Timotheos A, Benos A Degiannakis S, 2003)

\section{Método de Cuasi-verosimilitud}

Sea el siguiente vector aleatorio $\mathrm{y}_{t} q u e s a t i s f a c e l a s i g u i e n t e e c u a c i n: y_{t}=g_{t}(\theta)+\epsilon_{t}(13)$

donde $\epsilon_{t}$ es una secuencia marginal diferenciada con respecto a $F_{t}$, donde $F_{t}$ la sigma álgebra comprendida por las observaciones de $y_{t}$ para $\mathrm{t}>1$ tal que $E\left(\epsilon_{t} \mid F_{t-1}\right)=E_{t-1}\left(\epsilon_{t}\right)$ y $g_{t}(\theta)$ corresponde a un $F_{t-1}$ medible siendo función de un vector de parámetros de interes $\theta$.

Se tiene que $E_{t-1}\left(\epsilon_{t} \epsilon_{t}^{\prime}\right)=A_{t}$ conocida, por lo que una función lineal para la estimación se escribe como:

$$
\mathbb{G}_{T}=\sum_{t=1}^{T} W_{t}\left(y_{t}-g_{t}(\theta)\right)
$$


con la siguiente función de quasi-verosimilitud

$$
G_{T}(\theta)=\sum_{t=1}^{T} \frac{\partial g_{t}(\theta)}{\partial \theta} A_{t}^{-1}\left(y_{t}-g_{t}(\theta)\right)
$$

donde $W_{t}$ es una matriz medible en $F_{t-1}$. Seguido a ello, la estimación para $\theta$ es la solución de la anterior ecuación cuando esta es igual a 0. Si sub estimación de los espacios de $G_{T}(\theta)$ es $\mathbb{G}_{t}=W_{t}\left(y_{t}-g_{t}(\theta)\right)$ entonces la función de cuasi-verosimilitud estimada es la siguiente:

$$
G_{(t)}(\theta)=\frac{\partial g_{t}(\theta)}{\partial \theta} A_{t}^{-1}\left(y_{t}-g_{t}(\theta)\right)
$$

y la estimación de $\theta$ eslasolucinde $\mathrm{G}_{T}(\theta)=0$

Para el uso del método de cuasi-verosimilitud en el caso del proceso GARCH (p,q), se tienen que tener en cuenta algunas consideraciones. Sea un proceso GARCH (p,q) definido de la siguiente manera:

$$
y_{t}=\mu+\varepsilon_{t}, \quad t=1,2, \ldots, T
$$

y

$$
\sigma_{t}^{2}=c_{0}+\sum_{i=1}^{q} c_{i} \varepsilon_{t-i}^{2}+\sum_{d=1}^{p} \beta_{d} \sigma_{t-d}^{2}+\zeta_{t}, \quad t=1,2, \ldots, T
$$

donde $\varepsilon_{t}$ son independientes e idénticamente distribuidos (i.i.d) con $E_{t-1}\left(\varepsilon_{t}\right)=0 \mathrm{y}$ $V A R_{t-1}\left(\varepsilon_{t}\right)=\sigma_{t}^{2}$;

$$
\zeta_{t}
$$

(i.i.d) con $E_{t-1}\left(\zeta_{t}\right)=0$ y $V A R_{t-1}\left(\zeta_{t}\right)=\sigma_{\zeta}^{2}$. En este caso para la siguiente diferencia:

$$
\left(\begin{array}{c}
\varepsilon_{t} \\
\zeta_{t}
\end{array}\right)=\left(\begin{array}{c}
y_{t}-\mu \\
\sigma_{t}^{2}-c_{0}-\sum_{i=1}^{q} c_{i} \varepsilon_{t-i}^{2}-\sum_{d=1}^{p} \beta_{d} \sigma_{t-d}^{2}
\end{array}\right)
$$

permite la estimación por (QL) para $\sigma_{t}^{2}$ como:

$$
G_{(t)}\left(\sigma_{t}^{2}\right)=(0,1)\left(\begin{array}{cc}
\sigma_{t}^{2} & 0 \\
0 & \sigma_{\zeta}^{2}
\end{array}\right)^{-1}\left(\begin{array}{l}
\varepsilon_{t} \\
\zeta_{t}
\end{array}\right)=\sigma_{\zeta}^{2}\left(\sigma_{t}^{2}-c_{0}-\sum_{i=1}^{q} c_{i} \varepsilon_{t-i}^{2}-\sum_{d=1}^{p} \beta_{d} \sigma_{t-d}^{2}\right)
$$

Entonces dando el valor de 0 en el tiempo 0 para $\varepsilon_{t}$, es decir $\widehat{\varepsilon_{0}}=0$, unos valores iniciales para la la estimación de los parámetros del modelo $\psi_{0}=\left(\mu_{0}, c_{00}, c_{10}, \ldots, c_{p_{0}}, \beta_{1_{0}}, \ldots, \beta_{q_{0}}, \sigma_{\zeta_{0}}^{2}\right)$, a estimación de los errores al cuadrado $\hat{\varepsilon}_{t-i}^{2}=\left(y_{t-i}-\mu_{0}\right)^{2}, \hat{\sigma}_{t-j}^{2}$ es la estimación por cuasi-verosimilitud de la parte de heterocedasticidad del modelo. Entonces la estimación por QL para $\sigma_{t}^{2}$ es la solucion de $G_{(t)}\left(\sigma_{t}^{2}\right)=0$.

$$
\hat{\sigma}_{t}^{2}=c_{0}+\sum_{i=1}^{q} c_{i} \hat{\varepsilon}_{t-i}^{2}+\sum_{d=1}^{p} \beta_{d} \hat{\sigma}_{t-d}^{2}
$$

El estimador QL usando la estimación para $\sigma_{t}^{2}$ y el vector de información $y_{t}$, para la estimación de $\theta=\left(\mu, c_{0}, c_{1}, \ldots, c_{q}, \beta_{1}, \ldots, \beta_{q}\right)$ esta dada por:

$$
G_{T}(\theta)=\sum_{t=1}^{T}\left(\begin{array}{cc}
-1 & 0 \\
0 & -1 \\
0 & -\varepsilon_{t-1}^{2} \\
\vdots & \vdots \\
0 & -\varepsilon_{t-p}^{2} \\
0 & -\sigma_{t-1}^{2} \\
\vdots & \vdots \\
0 & -\sigma_{t-p}^{2}
\end{array}\right)\left(\begin{array}{cc}
\sigma_{t}^{2} & 0 \\
0 & \sigma_{\zeta_{0}}^{2}
\end{array}\right)^{-1}\left(\begin{array}{c}
\varepsilon_{t} \\
\zeta_{t}
\end{array}\right)
$$


El estimador para $\theta$ correspondealasolucinde $\mathrm{G}_{T}(\theta)=0$. Donde $\widehat{\zeta}_{t}$ y $\hat{\sigma}_{\zeta}^{2}$ son:

$$
\widehat{\zeta}_{t}=\hat{\sigma}_{t}^{2}-\left(c_{0}+\sum_{i=1}^{q} c_{i} \hat{\varepsilon}_{t-i}^{2}+\sum_{d=1}^{p} \beta_{d} \hat{\sigma}_{t-d}^{2}\right) \hat{\sigma}_{\zeta}^{2}=\left(\sum_{t=1}^{T}\left(\widehat{\zeta}_{t}-\overline{\widehat{\zeta}}_{t}\right)^{2}\right) T-1
$$

Entonces la estimación de los parámetros por cuasi-verosimilitud $\hat{\psi}=\left(\hat{\mu}, \widehat{c_{0}}, \widehat{c_{1}}, \ldots, \widehat{c_{p}}, \widehat{\beta_{1}}, \ldots, \widehat{\beta_{p}}, \hat{\sigma}_{\zeta}^{2}\right)$ corresponde a una iteración de las anteriores funciones a partir del valor inicial para $\psi_{0}$.

En el caso del modelo $\operatorname{GARCH}(1,1)$ se tiene en cuenta la siguiente forma para la varianza $\sigma_{t}^{2}$.

$$
\sigma_{t}^{2}=c_{0}+c_{1} \varepsilon_{t-1}^{2}+\beta_{1} \sigma_{t-1}^{2}+\zeta_{t}
$$

donde $\varepsilon_{t}(i . i . d) ; E_{t-1}\left(\varepsilon_{t}\right)=0, V A R_{t-1}\left(\varepsilon_{t}\right)=\sigma_{t}^{2} \mathrm{y} \zeta_{t}$ (i.i.d) con $E_{t-1}\left(\zeta_{t}\right)=0 \mathrm{y}$ $V A R_{t-1}\left(\zeta_{t}\right)=\sigma_{\zeta}^{2}$. En este caso la diferencia

$$
\left(\begin{array}{c}
\varepsilon_{t} \\
\zeta_{t}
\end{array}\right)=\left(\begin{array}{c}
y_{t}-\mu \\
\sigma_{t}^{2}-\left(c_{0}+c_{1} \varepsilon_{t-1}^{2}+\beta_{1} \sigma_{t-1}^{2}+\zeta_{t}\right)
\end{array}\right)
$$

Y el estimador de cuasi-verosimilitud para $\sigma_{t}^{2}$ esta dado por: $G_{(t)}\left(\sigma_{t}^{2}\right)=$

$$
(0,1)\left(\begin{array}{cc}
\sigma_{t}^{2} & 0 \\
0 & \sigma_{\zeta}^{2}
\end{array}\right)^{-1}\left(\begin{array}{c}
y_{t}-\mu \\
\sigma_{t}^{2}-\left(c_{0}+c_{1} \varepsilon_{t-1}^{2}+\beta_{1} \sigma_{t-1}^{2}+\zeta_{t}\right)
\end{array}\right)
$$

En este caso dando valores iniciales para $\widehat{\varepsilon_{0}}=0, \psi_{0}=\left(\mu_{0}, c_{00}, c_{10}, \beta_{10}, \sigma_{\zeta_{0}}^{2}\right), \hat{\varepsilon}_{t-1}^{2}=$ $\left(y_{t-1}-\mu_{0}\right)^{2}$ y $\hat{\sigma}_{t-1}^{2}$ es el estimador por cuasi-verosimilitud para $\sigma_{t-1}^{2}$, por lo que la solución de $G_{(t)}\left(\sigma_{t}^{2}\right)=0$ resulta ser el estimador $G_{T}(\theta)$ para la varianza $\hat{\sigma}_{t}^{2}$ con la forma:

$$
\begin{gathered}
\hat{\sigma}_{t}^{2}=c_{0}+c_{1} \hat{\varepsilon}_{t-1}^{2}+\beta_{1} \hat{\sigma}_{t-1}^{2} \\
G_{T}\left(\mu, c_{0}, c_{1}, \beta_{1}\right)=\sum_{t=1}^{T}\left(\begin{array}{cc}
-1 & 0 \\
0 & -1 \\
0 & -\varepsilon_{t-1}^{2} \\
0 & -\sigma_{t-1}^{2}
\end{array}\right)\left(\begin{array}{cc}
\sigma_{t}^{2} & 0 \\
0 & \sigma_{\zeta_{0}}^{2}
\end{array}\right)^{-1}\left(\begin{array}{c}
y_{t}-\mu \\
\sigma_{t}^{2}-\left(c_{0}+c_{1} \varepsilon_{t-1}^{2}+\beta_{1} \sigma_{t-1}^{2}+\zeta_{t}\right)
\end{array}\right)
\end{gathered}
$$

la solución $G_{T}\left(\mu, c_{0}, c_{1}, \beta_{1}\right)=0$, permite la estimación de los parámetros con la siguiente forma (Alzghool, 2017):

$$
\begin{aligned}
& \hat{\mu}=\frac{\sum_{t=1}^{T} \frac{y t}{\hat{\sigma}_{t}^{2}}}{\sum_{t=1}^{T} \frac{1}{\hat{\sigma}_{t}^{2}}} \\
& \hat{\beta}_{1}=\frac{\Delta_{\hat{\sigma}_{t-1}^{2} \hat{\varepsilon}_{t-1}^{2}} \Delta_{\hat{\sigma}_{t}^{2} \hat{\varepsilon}_{t-1}^{2}}-\Delta_{\hat{\varepsilon}_{t-1}^{2} \hat{\varepsilon}_{t-1}^{2}} \Delta_{\hat{\sigma}_{t}^{2} \hat{\sigma}_{t-1}^{2}}}{\Delta_{\hat{\sigma}_{t-1}^{2}}^{2} \hat{\varepsilon}_{t-1}^{2}-\Delta_{\hat{\sigma}_{t-1}^{2}} \hat{\sigma}_{t-1}^{2} \Delta_{\hat{\varepsilon}_{t-1}^{2}} \hat{\varepsilon}_{t-1}^{2}} \\
& \widehat{c_{0}}=\frac{\sum_{t=1}^{T} \hat{\sigma}_{t}^{2}-\widehat{c}_{1} \sum_{t=1}^{T} \hat{\varepsilon}_{t-1}^{2}-\hat{\beta}_{1} \sum_{t=1}^{T} \hat{\sigma}_{t-1}^{2}}{T} \quad \mathrm{y} \\
& \widehat{c_{1}}=\frac{\Delta_{\hat{\sigma}_{t}^{2} \hat{\varepsilon}_{t-1}^{2}}-\hat{\beta}_{1} \Delta_{\hat{\sigma}_{t-1}^{2} \hat{\varepsilon}_{t-1}^{2}}}{\Delta_{\hat{\varepsilon}_{t-1}^{2} \hat{\varepsilon}_{t-1}^{2}}}
\end{aligned}
$$


donde:

$$
\begin{gathered}
\Delta_{\hat{\sigma}_{t-1}^{2} \hat{\varepsilon}_{t-1}^{2}}=\sum_{t=1}^{T} \hat{\sigma}_{t-1}^{2} \hat{\varepsilon}_{t-1}^{2}-\frac{\sum_{t=1}^{T} \hat{\sigma}_{t-1}^{2} \sum_{t=1}^{T} \hat{\varepsilon}_{t-1}^{2}}{T} \\
\Delta_{\hat{\sigma}_{t}^{2} \hat{\varepsilon}_{t-1}^{2}}=\sum_{t=1}^{T} \hat{\sigma}_{t}^{2} \hat{\varepsilon}_{t-1}^{2}-\frac{\sum_{t=1}^{T} \hat{\sigma}_{t}^{2} \sum_{t=1}^{T} \hat{\varepsilon}_{t-1}^{2}}{T} \\
\Delta_{\hat{\varepsilon}_{t-1}^{2} \hat{\varepsilon}_{t-1}^{2}}=\sum_{t=1}^{T} \hat{\varepsilon}_{t-1}^{4}-\frac{\left(\sum_{t=1}^{T} \hat{\varepsilon}_{t-1}^{2}\right)^{2}}{T} \\
\Delta_{\hat{\sigma}_{t}^{2} \hat{\sigma}_{t-1}^{2}}=\sum_{t=1}^{T} \hat{\sigma}_{t}^{2} \hat{\sigma}_{t-1}^{2}-\frac{\sum_{t=1}^{T} \hat{\sigma}_{t}^{2} \sum_{t=1}^{T} \hat{\sigma}_{t-1}^{2}}{T} \\
\Delta_{\hat{\sigma}_{t-1}^{2} \hat{\sigma}_{t-1}^{2}}=\sum_{t=1}^{T} \hat{\sigma}_{t}^{4}-\frac{\left(\sum_{t=1}^{T} \hat{\sigma}_{t-1}^{2}\right)^{2}}{T}
\end{gathered}
$$

Las estimaciones se obtienen con el anterior procedimiento iterativo tomando en consideración los valores iniciales para $\psi_{0}=\left(\mu_{0}, c_{00}, c_{10}, \beta_{10}, \sigma_{\zeta_{0}}^{2}\right)$.

$\mathrm{Al}$ considerar la anterior estimación de la varianza para el modelo GARCH se encuentra el cuantil $(1-\alpha)$ teniendo en cuenta la siguiente fomra:

$$
\operatorname{VaR} R_{T}^{\alpha}=\text { quantile }\left[S\left(\hat{\sigma}_{t}^{2} * Z_{\alpha}+\hat{r}_{t}\right)\right]
$$

En donde $S=1, \hat{\sigma}_{t}^{2}$ la varianza estimada del modelo, $Z_{\alpha}$ el cuantil de la normal a un $\alpha$ y $\hat{r}_{t}$ los rendimientos estimados por el modelo en el tiempo t.

\subsection{Estimación del VaR con el uso de Cópulas}

Teniendo en cuenta la volatilidad de las series financieras, en la mayoría de los casos se asume normalidad sobre los retornos, con estos supuestos se realizan los modelos GARCH; sin embargo, es clave entender la relación de dependencia no lineal existente entre los retornos por medio de la teoría de cópulas. Una cópula es entendida como la relación entre dos o mas funciones, la cual denota un vínculo entre la distribución de probabilidad de un vector aleatorio y las distribuciones marginales asociadas a este. En términos multivariados una cópula se puede expresar como:

$$
H\left(x_{1}, x_{2}, \ldots, x_{d}\right)=C\left(F_{x_{1}}, F_{x_{2}}, \ldots, F_{x_{3}}\right)
$$

donde $C$ denota esta relación y puede ser escrita como:

$$
C\left(F_{1}\left(x_{1}\right), F_{2}\left(x_{2}\right), \ldots, F_{d}\left(x_{d}\right)\right)=P\left(U_{1} \leq u_{1}, U_{2} \leq u_{2}, \ldots, U_{d} \leq u_{d}\right)
$$

En finanzas, la aplicación de las cópulas ha ido creciendo en los últimos años, debido a su uso en proceso matemáticos de gran complejidad.

Formalmente las cópulas son funciones multivariadas d-dimensionales con distribuciones marginales en el intervalo [0,1]; éstas, son usadas para el modelado de la estructura de dependencia en datos multivariados, donde $X=\left(X_{1}, X_{2}, \ldots, X_{d}\right)$ corresponde a un vector d-dimensional con una distribución conjunta $F\left(x_{1}, \ldots, x_{d}\right)$ y distribuciones marginales $F_{i}\left(x_{i}\right), i=1,2, \ldots, d$. tal que:

$$
F\left(x_{1}, \ldots, x_{d}\right)=C\left(F_{1}\left(x_{1}\right), F_{2}\left(x_{2}\right), \ldots, F_{d}\left(x_{d}\right)\right)
$$

En donde la anterior expresión satisface las siguientes propiedades (Alzghool, 2017):

- Si $F$ es una función continua d-variada con marginales univariadas $F_{1}, \ldots, F_{d} y F_{1}^{-1}, \ldots, F_{d}^{-1}$, entonces:

$C\left(F_{1}\left(x_{1}\right), F_{2}\left(x_{2}\right), \ldots, F_{d}\left(x_{d}\right)\right)=F\left(F_{1}^{-1}\left(u_{1}\right), F_{2}^{-1}\left(u_{2}\right), \ldots, F_{d}^{-1}\left(u_{d}\right)\right), \quad u \in[0,1]^{d}$

es única 
REMEF (The Mexican Journal of Economics and Finance) Análisis comparativo de las metodologías de estimación semiparamétricas

- Si F es una función continua d-variada de variables discretas (generalmente mitad continua, mitad discreta), entonces la cópula es unica en el conjunto

$$
\operatorname{rango}\left(F_{1}\right) \times \cdots \times \operatorname{rango}\left(F_{d}\right)
$$

Entonces, según (Joe, 2015), para cualquier función de distribución cuyas marginales sean uniformes, es posible obtener una cópula asociada. En la función cópula $C$ de un vector multivariado $X$ se encuentra la función de distribución del vector $U=\left(U_{1}, U_{2}, \ldots, U_{d}\right)^{t}$ donde, $F_{i}$ es la distribución marginal de cada variable aleatoria, lo que implica que:

$$
H\left(x_{1}, x_{2}, \ldots, x_{d}\right)=C\left(F_{x_{1}}, F_{x_{2}}, \ldots, F_{x_{3}}\right)
$$

donde $H$ es la función de distribución conjunta de $x_{1}, x_{2}, \ldots, x_{d}$ en el cubo uniforme $I^{n}$. En probabilidad, una cópula $C:[0,1]^{d} \rightarrow[0,1]$ es d-dimensional si $C$ es una distribución de probabilidad conjunta de un vector d-dimensional dentro del cubo uniforme constituido por los intervalos $[0,1]^{d}$ con sus marginales uniformes.

\section{1) Teorema de Sklar}

De acuerdo con el teorema de Sklar, se establece que si en $H\left(x_{1}, x_{2}, \ldots, x_{d}\right)$ las distribuciones $F_{i}$ son continuas entonces cópula asociada $\mathrm{C}$ es única. Si no, entonces esta resulta ser en el producto cruzado entre los rangos de cada $F_{i}$ y es unica para $H$.

\section{2) Vine Cópulas}

La implicación del teorema de Sklar muestra que las marginales uniformes pueden ser modeladas de manera separada del modelado de dependencia en términos de la cópula, mostrando algunos problemas de selección en la estructura de dependencia en altas dimensiones. Las cópulas multivariadas como la Gaussiana o t de student son definidas para cualquier dimension; sin embargo, sus propiedades de dependencia suelen presentar limitaciones y pueden presentar dificultades incluso en tres dimensiones. En el caso de las arquimedianas presentan poca flexibilidad en modelar la dependencia con dos o más variables (Brechmann \& Schepsmeier, 2013).

Este problema puede ser solucionado con el uso de Vine Cópulas; este método permite escribir una distribución conjunta en términos de una cópula bivariada y una condicional conforme a una estructura gráfica en forma de árbol, por medio de esta permite una medida más flexible para capturar la estructura de dependencia entre los activos financieros. Existen dos clases de Vine Cópulas, los C-Vinces (canonicos) \& D-Vines. En estos, la diferencia se basa en la forma estructural del árbol en el que se representan las cópulas condicionales y bivariadas. Estas estructuras han sido utilizadas satisfactoriamente en distintas aplicaciones, siendo la mas usada el riesgo financiero. Las Vine Cópulas están conformadas por una estructura general hecha a partir de cópulas bivariadas o llamadas (Pair Cópulas Constructions PCC), esto permite que una cópula general pueda ser reescrita en un producto entre PCC usando las distribuciones multivariadas con unas marginales dadas.

Sea $X$ un vector aleatorio multivariado con $F_{i}$ distribuciones marginales, $f\left(x_{1}, x_{2}, \ldots, x_{d}\right)$ una función de densidad d-dimensional y $c\left(u_{1}, u_{2}, \ldots, u_{d}\right)$ la correspondiente densidad cópula. De acuerdo con lo mencionado por (Czado, C. Aleksey. M, 2010)., la función de distribución conjunta $f\left(x_{1}, x_{2}, \ldots, x_{d}\right)$ puede ser expresada en términos de una distribución condicional como se presenta a continuación:

$$
\begin{gathered}
f\left(x_{1}, x_{2}, \ldots, x_{d}\right)=\prod_{k=1}^{d} f_{k}\left(x_{k}\right) \times \prod_{i=1}^{d-1} \prod_{j=1}^{d-i} c_{j, j+i \mid(i+1):(i+j-1)} \\
\left(F\left(x_{j} \mid x_{j+1}, \ldots, x_{j+i-1}\right), F\left(x_{i+j} \mid x_{j+1}, \ldots, x_{j+i-1}\right), \theta_{i, i+j \mid(i+j):(i+j-1)}\right)
\end{gathered}
$$

donde $c_{i, i+j \mid(i+j):(i+j-1)}$ corresponden a las densidades de las cópulas bivariadas con los parametros $\theta_{i, i+j \mid(i+j):(i+j-1)}$. En este trabajo se hara el uso de la descomposición D-vine la cual es construida seleccionando un orden especifico de las variables. En esta 
estructura los órdenes de relación entre los activos financieros son escritos de tal manera en que cada par condicional encontrado permita hallar una nueva relación condicional entre las relaciones previamente calculadas.

Siguiendo con los delineamientos de este trabajo, se tienen tres dimensiones conformadas por los activos con mayor peso en el portafolio, por lo que una posible descomposición para la densidad $f\left(x_{1}, x_{2}, \ldots, x_{d}\right)$ puede ser:

$$
f\left(x_{1}, x_{2}, x_{3}\right)=f_{3 \mid 12}\left(x_{3} \mid x_{1}, x_{2}\right) f_{2 \mid 1}\left(x_{2} \mid x_{1}\right) f_{1}\left(x_{1}\right)
$$

lo que implica que

$$
\begin{gathered}
f_{2 \mid 1}\left(x_{2} \mid x_{1}\right)=c_{12}\left(F_{1}\left(x_{1}\right), F_{2}\left(x_{2}\right)\right) f_{1}\left(x_{1}\right) f_{3 \mid 12}\left(x_{3} \mid x_{1}, x_{2}\right)= \\
c_{3 \mid 12}\left(F_{1 \mid 2}\left(x_{1} \mid x_{2}\right) F_{2 \mid 3}\left(x_{2} \mid x_{3}\right)\right) f_{3 \mid 2}\left(x_{3} \mid x_{2}\right) f_{3 \mid 2}\left(x_{3} \mid x_{2}\right)=c_{23}\left(F_{2}\left(x_{2}\right), F_{3}\left(x_{3}\right)\right) f_{3}\left(x_{3}\right)
\end{gathered}
$$

por lo que la descomposición para $\mathrm{d}=3$ es:

$$
\begin{gathered}
f\left(x_{1}, x_{2}, x_{3}\right)=f_{1}\left(x_{1}\right) f_{2}\left(x_{2}\right) f_{3}\left(x_{3}\right) \cdot c_{12}\left(F_{1}\left(x_{1}\right), F_{2}\left(x_{2}\right)\right) c_{23}\left(F_{2}\left(x_{2}\right),\right. \\
F_{3}\left(x_{3}\right) \cdot c_{3 \mid 12}\left(F_{1 \mid 2}\left(x_{1} \mid x_{2}\right) F_{2 \mid 3}\left(x_{2} \mid x_{3}\right)\right)
\end{gathered}
$$

esto implica que la estructura gráfica del árbol D-vine tiene la siguiente forma:

Figura 1. Estructura del árbol D-Vine

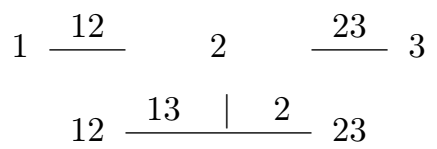

Fuente: Elaboración propia

Lo que significa que la cópula general para esta estructura resulta del producto entre las PCC como se muestra a continuación:

$$
c\left(u_{1}, u_{2}, u_{3}\right)=c_{12} \cdot c_{13} \cdot c_{13 \mid 2}
$$

\section{Familia de cópulas}

A continuación, se muestran las posibles cópulas que pueden obtenerse en el modelado de los activos financieros (Nicole Krämer Ulf Schepsmeier, 2011):

\section{Cópula Gaussiana}

Se define a la cópula gaussiana como la distribución sobre el espacio medible en el cubo $[0,1]^{d}$ construida a partir de la Normal multivariada y dada una matriz de correlación $\Sigma \in \mathbb{R}^{d}$ donde la relación d-dimensional se escribe de la siguiente manera:

$$
C(u)=\Phi_{\Sigma}\left(\Phi^{-1}\left(u_{1}\right), \ldots, \Phi^{-1}\left(u_{d}\right)\right)
$$

donde $\Phi^{-1}$ es la inversa de la distribución normal estándar $\Phi_{\Sigma}$; en términos matemáticos la densidad de la cópula se puede escribir como:

$$
C(u)=\frac{1}{\sqrt{\operatorname{det} \Sigma}}\left(-\frac{1}{2}\left(\begin{array}{c}
\Phi^{-1}\left(u_{1}\right) \\
\vdots \\
\Phi^{-1}\left(u_{d}\right)
\end{array}\right) \cdot\left(\Sigma^{-1}-I\right) \cdot\left(\begin{array}{c}
\Phi^{-1}\left(u_{1}\right) \\
\vdots \\
\Phi^{-1}\left(u_{d}\right)
\end{array}\right)\right)
$$




\section{Cópula $t$}

En términos generales la cópula t puede ser escrita como:

$$
C_{v, \rho}^{t}(u)=\frac{f_{v, P}\left(t_{v}^{-1}\left(u_{1}\right), \ldots, t_{v}^{-1}\left(u_{d}\right)\right)}{\prod_{i=1}^{d} f_{v}\left(t_{v}^{-1}\left(u_{i}\right)\right)}
$$

donde $f_{v, P}$ es la densidad de un vector distribuido $t_{d}(v, 0, P)$ con $\mathrm{v}$ grados de libertad y $\mathrm{P}$ como matriz de correlaciones, y $f_{v}$ como densidad univariada estándar $\mathrm{t}$ con $\mathrm{v}$ grados de libertad (Nelsen, 2006)

\section{Cópulas arquimedianas}

Las cópulas arquimedianas son una clase asociativa de cópulas cuya expresion admite una fórmula explícita; estas son populares por permitir modelar la dependencia en dimensiones altas con un solo parámetro. En general, una cópula se dice arquimediana si se expresa como:

$$
C\left(u_{1}, \ldots, u_{d} ; \theta\right)=\psi^{[-1]}\left(\psi\left(u_{1} ; \theta\right)+\cdots+\psi\left(u_{d} ; \theta\right) ; \theta\right)
$$

donde $\psi$ es una función decreciente, continua y convexa perteneciente al intervalo $[0,1] \times \Theta \rightarrow[0, \infty), \theta$ es el parámetro de la cópula con un espacio de parámetros $\Theta y \psi^{[-1]}$ es considerado como pseudo inversa de $\psi$ tal que:

$$
\psi^{[-1]}(t ; \theta)=\left\{\begin{array}{l}
\psi^{-1}(t ; \theta) \text { si } 0 \leq t \leq \psi(0 ; \theta) \\
0 \quad \text { si } \psi(0 ; \theta) \leq t \leq \infty
\end{array}\right.
$$

En general las cópulas arquimedianas más importantes son las siguientes:

Tabla 1. Cópulas Arquimedianas

\begin{tabular}{|l|c|c|}
\hline \multicolumn{1}{|c|}{ Cópula } & Función de distribución & Parámetro \\
\hline Ali-Mikhail-Haq & $\frac{u v}{1-\theta(1-u)(1-v)}$ & $\theta \in[-1,1)$ \\
\hline Clayton & {$\left[\operatorname{máx}\left\{u^{-\theta}+v^{-\theta}-1\right\} ; 0\right]^{-1 / \theta}$} & $\theta \in[-1, \infty) / 0$ \\
\hline Frank & $-\frac{1}{\theta} \log \left[1+\frac{(\exp (-\theta u)-1)(\exp (-\theta v)-1)}{(\exp (-\theta)-1)}\right]$ & $\theta \in(\mathbb{R}) / 0$ \\
\hline Gumbel & $\exp \left[-\left((1-\log (u))^{\theta}+(1-\log (v))^{\theta}\right)\right]$ & $\theta \in[-1, \infty)$ \\
\hline Joe & $1-\left[(1-u)^{\theta}+(1-v)^{\theta}-(1-u)^{\theta}(1-v)^{\theta}\right]^{1 / \theta}$ & $\theta \in[-1, \infty)$ \\
\hline
\end{tabular}

Fuente: (Nelsen, 2006) y (Triana, Torres Aponte, Alba, Pineda-Ríos, 2017)

Estimación de los parámetros

En general un modelo con la inclusión de una estructura de dependencia (Vine Cópula) posee la siguiente forma:

$$
\text { Modelo }=\text { Estructura }(\text { arbol })+\text { copula }+ \text { parametros }
$$

La selección de la estructura es utilizada por medio de el cálculo del $\tau$ de kendall, el $\rho$ de spearman, $\mathrm{p}$ valores significativos en las pruebas de bondad y ajuste o distancias, en donde el árbol es seleccionado como una estructura canónica $\mathrm{C}$-vine, D-vine o R-vine en general.

Los métodos de selección para la cópula a utilizar se basan en test de bondad y ajuste, independencia, criterios como Akaike Information Criterion (AIC) o Bayesian Information Criterion (BIC), o gráficos de contorno que permitan identificar una posible estructura.

En cuanto a la última parte del modelo, la estimación de los parámetros se puede realizar por tres métodos en concreto (Triana, Torres Aponte, Alba, Pineda-Ríos, 2017):

\section{- Estimación por máxima verosimilitud}


- Métodos secuenciales: En estos los parámetros son secuencialmente estimados desde el inicio de la estructura del árbol, generando así pseudo observaciones usadas en la segunda etapa del árbol, en donde esta estimación se realiza bajo máxima verosimilitud bivariada o $\theta=f(\tau)$. En este método las estimaciones secuenciales pueden ser utilizadas como valores iniciales para la máxima verosimilitud.

\section{- Estimación Bayesiana}

En el caso de este trabajo la estimación de los parámetros se realizó por máxima verosimilitud por medio del paquete VineCópula del software R.

\subsection{Valor en Riesgo Condicional (Expected Shortfall)}

El VaR condicional (CVaR), déficit esperado o llamado expected shortfall es una medida del valor en riesgo que responde a la pregunta de "Si las cosas van mal que pérdidas se obtendrían". Desde un punto de vista estadístico en el CVaR se asume cada dia $t=$ $1, \ldots, T$, un $Y_{t}$ como las pérdidas y ganancias distribuidas a lo largo de una distribución real (incognoscible) $F_{t}$ pronosticada a partir de la distribución predictiva del modelo $P_{t}$ condicionada en la información previa para calcular el VaR. El valor en riesgo condicional puede ser definido como:

$$
E S_{t}^{\alpha}=\frac{1}{\alpha} \int_{0}^{\alpha} P_{t}^{-1}(q) d q
$$

Para las variables aleatorias $Y=Y_{t}$ se establece el supuesto de independencia más no el de ser idénticamente distribuidas (i.i.d), no se restringe la variabilidad de $F_{t}$ y $P_{t}$ en el tiempo y se asume que las distribuciones son continuas y crecientes, por lo que el el valor en riesgo condicional se puede escribir como (Alzghool, 2017):

$$
E S_{t}^{\alpha}=-E\left[Y_{t} \mid Y_{t}+\operatorname{VaR} R_{t}^{\alpha}<0\right]
$$

En términos generales el valor en riesgo condicional se expresa como el promedio de los $\alpha$ peores escenarios y puede ser calculado como el promedio de los días que se excede el VaR con un determinado nivel de confianza.

$$
E S_{t}^{1-\alpha}=E\left(r-V a R_{t}^{\alpha}\right)=\frac{1}{1-F_{r}\left(V_{a} R_{t}^{\alpha}\right)} \int_{U}^{V a R_{t}^{\alpha}} r_{1} f_{r}(r) d r
$$

donde $\mathrm{U}$ es el extremo inferior de los rendimientos y tanto $\mathrm{f}$ como $\mathrm{F}$ son funciones de densidad de la distribución de los rendimientos. Para el cálculo es necesario tomar en cuenta la siguiente expresión:

$$
E S_{t}^{\alpha}=C V a R_{t}^{\alpha}=\left(\mu-\sigma \cdot \frac{\phi\left(Z_{\alpha}\right)}{1-\Phi\left(Z_{\alpha}\right)}\right)
$$

donde $\mu, \sigma, \phi\left(Z_{\alpha}\right)$ y $\Phi\left(Z_{\alpha}\right)$ corresponden a la media, desviación estándar, valor en densidad y valor en distribución normal en el cuantil $1-\alpha$ de los rendimientos respectivamente.

\section{Medición del Valor en Riesgo en el mercado accionario colom- biano bajo metodologías semiparamétricas y Vine Cópulas}

El presente trabajo, es de carácter cuantitativo en el que se realizan las distintas técnicas de estimación para el valor en riesgo (VaR) a cada uno de los rendimientos de las acciones que conforman el índice accionario COLCAP; para tal efecto, se toman las acciones que mayor capitalización bursátil presentaron para el período 2015 a 2017: "Preferencial Bancolombia", "Grupo Sura", "Ecopetrol" y "Bancolombia". Una vez efectuados los cálculos se realizan estadísticos descriptivos y gráficos para identificar las características de las series 
financieras, debido al comportamiento leptocúrtico de los datos y la amplia volatilidad presentada. Con la ayuda del Software Eviews, se aplican métodos de estimación sobre las series con el fin de encontrar los mejores modelos que explique el comportamiento de los rendimientos de las acciones.

Una vez seleccionados los mejores modelos con el criterio AIC (Akaike information criterion), y realizando análisis de independencia y homocedasticidad de los residuales con una significancia del $10 \%(\alpha=0,1)$, los datos son exportados al software $\mathrm{R}$ donde se emplean técnicas para modelar la volatilidad esperada usando modelos de heterocedasticidad condicional ARCH-GARCH. Una vez realizado el modelo para capturar la volatilidad de los rendimientos, se procede a verificar la normalidad de los residuales con el fin de realizar las estimaciones en valor en riesgo por métodos semiparamétricos y valor en riesgo condicional para los rendimientos.

Finalmente, se construye un portafolio conformado por los rendimientos de las acciones citadas con el fin de estimar el valor en riesgo por medio de Cópulas; en este caso, se optó por una estructura D-Vine para el conjunto de rendimientos. Una vez identificada esta estructura, con los modelos GARCH previamente calculados, se realizó la estimación del valor en riesgo siguiendo la estructura de dependencia no lineal que el portafolio presenta.

\subsection{Estimación del Valor en Riesgo bajo metodologías semipara- métricas en el Mercado accionario colombiano período 2008-2016}

A continuación, se toman las acciones que mayor peso tienen el índice de COLCAP, las cuales representan el $34 \%$ del volumen de negociación en una jornada

\subsubsection{Rendimientos de la acción preferencial Bancolombia}

En la figura se puede observar visualmente falta de tendencia sobre los rendimientos de la acción preferencial de Bancolombia

Figura 2. Linea Rendimientos de la acción preferencial Bancolombia

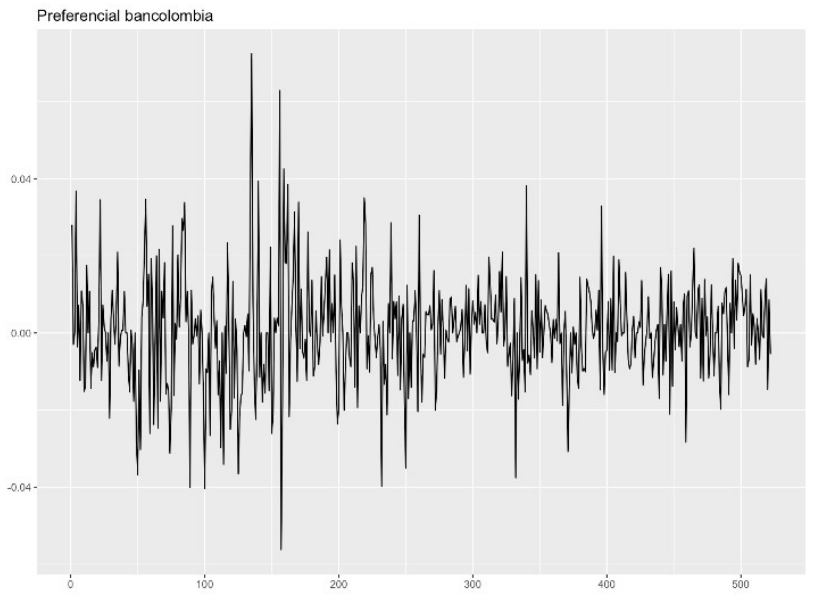

Fuente: Elaboración propia

Una vez identificado la falta de presencia de tendencia en la serie se realiza la prueba estadística de Phillips Perron con el fin de identificar de estacionariedad (presencia de raíz unitaria) en los rendimientos como se muestra a continuación: 
Tabla 2. Prueba raíz unitaria acción preferencia Bancolombia Null Hypothesis: PREFBAN has a unit root

Exogenous: Constant

Bandwidth: 8 (Newey-West automatic) using Bartlett Kernel

\begin{tabular}{|l|c|c|c|}
\hline \multirow{2}{*}{ Phillips-Perron test statistic } & Adj. t-Stat & Prob * \\
\cline { 3 - 3 } & & -22.89901 & 0.000 \\
\hline \multirow{3}{*}{ Test critical values } & $1 \%$ level & -3.442673 & \\
\cline { 2 - 3 } & $5 \%$ level & -2.866868 & \\
\cline { 2 - 3 } & $10 \%$ level & -2.569669 & \\
\hline
\end{tabular}

*Mackinnon (1996) one-sided p-values

Fuente: Elaboración propia

En la anterior tabla, se puede observar que se rechaza la hipótesis nula de ausencia de raíz unitaria en la serie, por lo que esta es estacionaria; debido a esto, se procede a la selección del mejor modelo en series de tiempo para los rendimientos "Preferencial Bancolombia" con el uso del siguiente correlograma:

Figura 3. Diagrama de Autocorrelación y autocorrelación parcial de la Acción preferencial Bamcolombia

\begin{tabular}{|c|c|c|c|c|c|c|}
\hline Autocorrelation & Partial Correlation & & $\mathrm{AC}$ & PAC & Q-Stat & Prob \\
\hline $1 \mid 1$ & $1 \mid 1$ & 1 & 0.002 & 0.002 & 0.0018 & 0.967 \\
\hline ודן & 1 & 2 & 0.050 & 0.050 & 1.3344 & 0.513 \\
\hline ין ו & ין ו & 3 & 0.034 & 0.034 & 1.9384 & 0.585 \\
\hline יןו & ון ו & 4 & 0.039 & 0.036 & 2.7337 & 0.603 \\
\hline וf & יקי & 5 & 0.042 & 0.039 & 3.6703 & 0.598 \\
\hline
\end{tabular}

Fuente: Elaboración propia

Para el caso de los rendimientos, se encontró que el modelo $A R M A(2,2)$ con un AIC de -5.656542 es el que mejor explica el comportamiento de los rendimientos. Siguiendo con el proceso, se hace un primer acercamiento a la distribución de los rendimientos, en donde, se verifica vía la prueba de Jarque-Bera la normalidad de los residuales como se visualiza a continuación:

Figura 4. Prueba de normalidad para el modelo de la acción preferencial Bancolombia

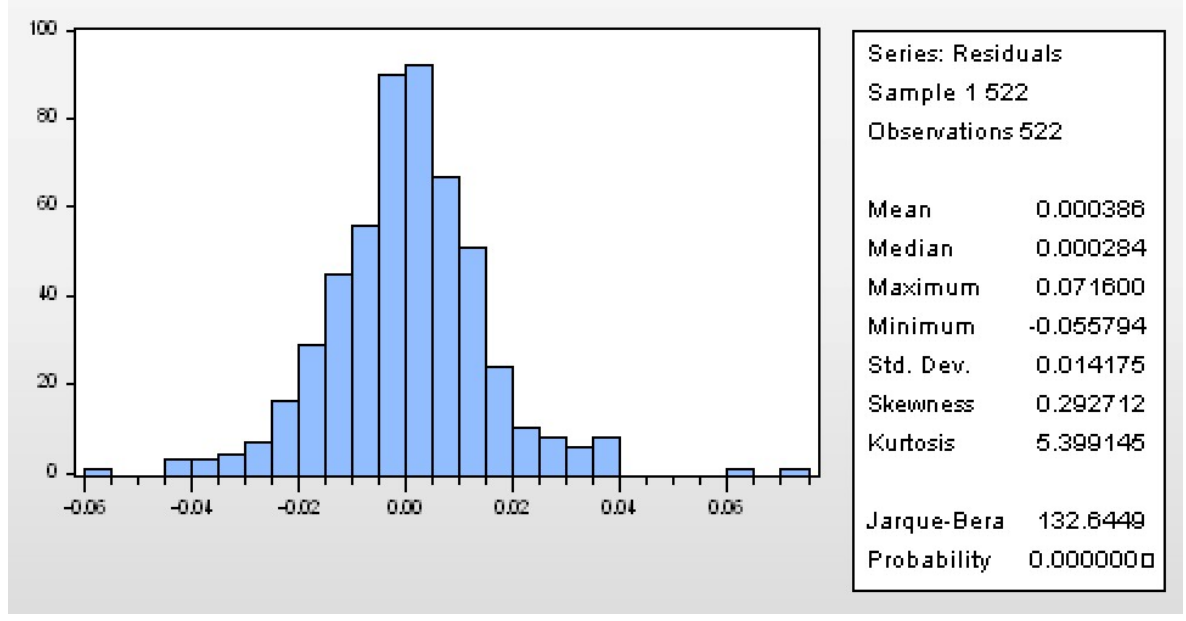

Fuente: Elaboración propia

Se puede observar en la figura 4, que se rechaza la hipótesis nula de normalidad para los residuales. Por último, en el modelado básico de la serie de tiempo se realiza la prueba 
REMEF (The Mexican Journal of Economics and Finance) Análisis comparativo de las metodologías de estimación semiparamétricas

de heterocedasticidad con el fin de validar el supuesto de volatilidad en los rendimientos de la serie como se muestra a continuación:

Tabla 3. Prueba de heterocedasticidad ARCH para los residuales de la acción preferencial Bancolombia

Heteroskedasticity: Test: ARCH

\begin{tabular}{|l|l|l|}
\hline F-statistic & 22.02460 & Prob. F (1,519) 0.0000 \\
\hline Obs*R-squared & 21.20942 & Prob. Chi-Square (1) 0.0000 \\
\hline \multicolumn{3}{|c|}{ Fuente: Elaboración propia }
\end{tabular}

De acuerdo con la tabla 3, se rechaza la hipótesis nula de homocedasticidad en los residuales por lo que existe la presencia de volatilidad. Siguiendo con el análisis, y considerando un nivel de significancia del $10 \%$, se puede concluir que las estimaciones del coeficiente del modelo de corto plazo del proceso en el modelo completo son significativas.

A continuación, se presenta el resultado de la prueba de Ljung-Box para los residuales del modelo identificado.

Tabla 4. Prueba de Ljung-Box sobre los residuales del modelo para la acción preferencial Bancolombia

\begin{tabular}{|c|c|c|}
\hline Q(lag) & Estadístico & p-value \\
\hline 1 & 0.6216 & 0.4304 \\
\hline 11 & 4.3726 & 0.9984 \\
\hline 19 & 7.3334 & 0.8760 \\
\hline \multicolumn{3}{|c|}{ Fuente: Elaboración propia }
\end{tabular}

Los resultados presentados en la tabla anterior, permiten concluir que no hay correlación serial en los residuales del modelo identificado. Lo anterior indica, que el modelo es adecuado para capturar la correlación serial a corto plazo presente en la serie.

Lo señalado anteriormente, es la prueba suficiente para la inclusión de los modelos GARCH en el análisis de la volatilidad del mercado y su efecto en la estimación del valor en riesgo.

A continuación, se observa el correlograma de los residuales al cuadrado:

Figura 5. Diagrama de autocorrelación y autocorrelación parcial de los residuales al cuadrado del modelo para la acción preferencial Bancolombia

fitpref@fit\$residuals
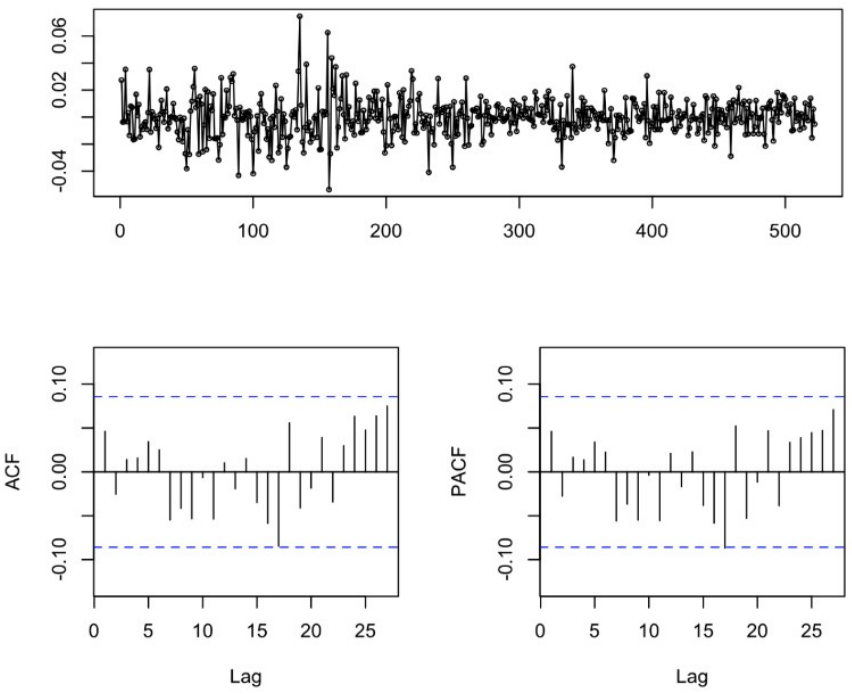

Fuente: Elaboración propia 
Se estableció que el modelo que mejor captura la volatilidad de los rendimientos lo constituye un $G A R C H(1,1)$ con un AIC de -5.789323 .

Teniendo en cuenta lo descrito anteriormente, se calcularon las siguientes estimaciones para el valor en riesgo:

Tabla 5. Estimaciones del VaR para la acción preferencial de Bancolombia

\begin{tabular}{|l|c|}
\hline \multicolumn{1}{|c|}{ Método de estimación } & Valor \\
\hline -Valor en Riesgo VaR & -0.0331548230 \\
\hline -Valor en riesgo condicional (CVaR) Expected Shortfall (ES) & -0.0375509408 \\
\hline -VaR - EVT/Distribución Gumbel & -0.0217651757 \\
\hline -VaR - EVT/Distribución generalizada del error & -0.0213318122 \\
\hline -Quasi-verosimilitud Fuente: Elaboración propia & -0.02004189 \\
\hline \multicolumn{2}{|c}{}
\end{tabular}

\subsubsection{Rendimientos de la acción Grupo Sura}

Se puede observar el posible comportamiento estacionario de los rendimientos en la siguiente figura:

Figura 6. Linea Rendimientos de la acción del Grupo Sura

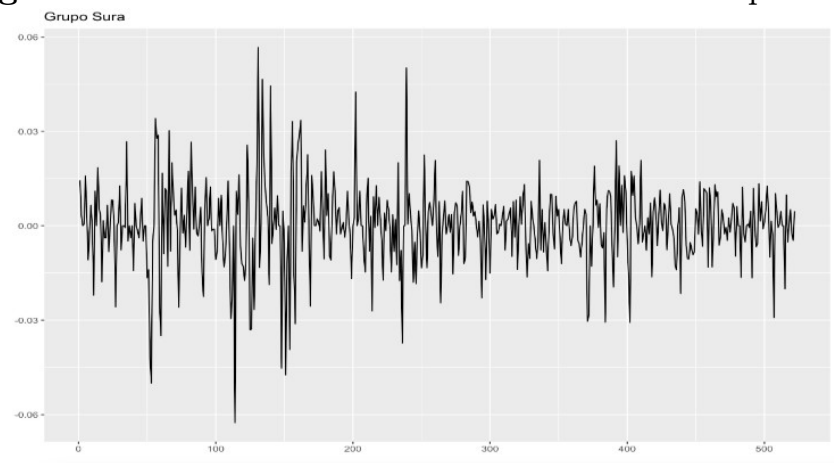

Fuente: Elaboración propia

De acuerdo con la figura 6 , se pudo identificar la falta de tendencia que presentan los rendimientos; ante este evento, se realiza la prueba estadística de Phillips Perron con el fin de identificar de estacionariedad (presencia de raíz unitaria) en los rendimientos como se muestra a continuación:

Tabla 6. Prueba raíz unitaria acción grupo Sura Null Hypothesis: GRUPOSURA has a unit root Exogenous: Constant Bandwidth: 14 (Newey-West automatic) using Bartlett Kernel

\begin{tabular}{|l|c|c|c|}
\hline \multirow{2}{*}{ Phillips-Perron test statistic } & Adj. t-Stat & Prob \\
\cline { 2 - 3 } & & -20.79134 & 0.000 \\
\hline \multirow{3}{*}{ Test critical values } & $1 \%$ level & -3.442673 & \\
\cline { 2 - 3 } & $5 \%$ level & -2.866868 & \\
\cline { 2 - 3 } & $10 \%$ level & -2.569669 & \\
\hline
\end{tabular}

*Mackinnon (1996) one-sided p-values Fuente: Elaboración propia

De acuerdo con la tabla 6, se puede observar que se rechaza la hipótesis nula de ausencia de raíz unitaria en la serie, por lo tanto, se deduce que es estacionaria. Debido a esto, se procede a la selección del mejor modelo en series de tiempo para los rendimientos "Grupo Sura" con el uso del siguiente correlograma: 
REMEF (The Mexican Journal of Economics and Finance) Análisis comparativo de las metodologías de estimación semiparamétricas y vía cópulas del Valor en Riesgo (VaR) en el mercado accionario colombiano

Figura 7. Diagrama de autocorrelación y autocorrelación parcial de la acción Grupo

\begin{tabular}{|c|c|c|c|c|c|}
\hline \\
\hline Autocorrelation & Partial Correlation & $\mathrm{AC}$ & PAC & Q-Stat & Prob \\
\hline 叹 & 吅 & 10.089 & 0.089 & 4.1584 & 0.041 \\
\hline 14 & 1 & $2-0.042$ & -0.051 & 5.1018 & 0.078 \\
\hline 10 & 10 1 & $3-0.034$ & -0.026 & 5.7205 & 0.126 \\
\hline 14 & 川1 & $4-0.028$ & -0.025 & 6.1271 & 0.190 \\
\hline d & di & $5-0.074$ & -0.073 & 9.0628 & 0.107 \\
\hline
\end{tabular}

Fuente: Elaboración propia

Para el caso de los rendimientos se encontró que el modelo $A R M A(2,1)$ con un AIC de -5.834519 era el que mejor explicaba el comportamiento de los rendimientos. Este cálculo es un primer acercamiento a la distribución de los rendimientos, el cual, se verifica con la prueba de Jarque-Bera, en donde se puede buscar detectar la normalidad de los residuales como se muestra a continuación:

Figura 8. Prueba de normalidad para el modelo de la acción grupo Sura

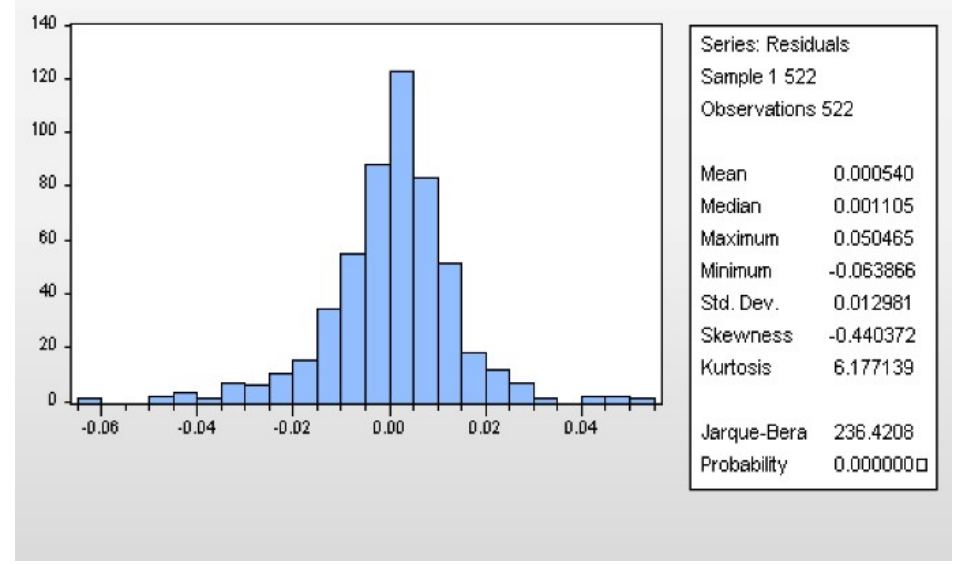

Fuente: Elaboración propia

Se puede observar en la figura 8 , que se rechaza la hipótesis nula de normalidad para los residuales.

Como último, en el modelado básico de la serie de tiempo, se realiza la prueba de heterocedasticidad con el fin de validar el supuesto de volatilidad en los rendimientos de la serie como se muestra a continuación:

Tabla 7. Prueba de heterocedasticidad ARCH para los residuales de la acción grupo Sura

Heteroskedasticity: Test: ARCH

\begin{tabular}{|l|l|l|}
\hline F-statistic & 3.736531 & Prob. F $(1,519) 0.0538$ \\
\hline Obs*R-squared & 3.724118 & Prob. Chi-Square (1) 0.0536 \\
\hline \multicolumn{3}{|c|}{ Fuente: Elaboración propia }
\end{tabular}

De acuerdo con la tabla 7, se rechaza la hipótesis nula de homocedasticidad en los residuales, por lo que deduce la presencia de volatilidad. Siguiendo con este desarrollo, y considerando un nivel de significancia del $10 \%$, se puede concluir que, las estimaciones del coeficiente del modelo de corto plazo del proceso en el modelo completo son significativas. 
En este aspecto, la prueba de Ljung-Box identificó que después del lag 14 con un pvalue del 0.366571 no existen problemas de correlación serial en los residuales del modelo identificado. Lo anterior indica, que el modelo es adecuado para capturar la correlación serial a corto plazo presente en la serie.

Lo dicho anteriormente, es la prueba suficiente para la inclusión de los modelos GARCH en el análisis de la volatilidad; para tal efecto, se realiza el diagrama de autocorrelación y autocorrelación parcial de los residuales al cuadrado:

Figura 9. Diagrama de autocorrelación y autocorrelación parcial de los residuales al cuadrado del modelo para la acción Grupo Sura

fitsura@fit\$residuals
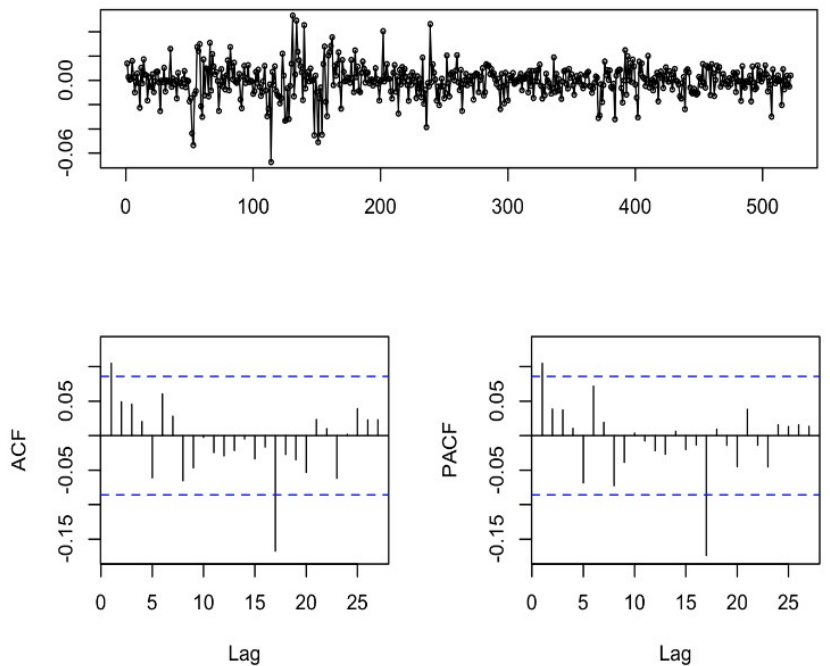

Fuente: Elaboración propia

De acuerdo con la figura 9, se estableció que, el modelo $G A R C H(1,1)$ con un AIC de -6.087352 es que mejor captura la volatilidad de los rendimientos.

Teniendo en cuenta lo descrito anteriormente, se calcularon las siguientes estimaciones para el valor en riesgo:

Tabla 8. Estimación del VaR para la acción de Grupo Sura

\begin{tabular}{|l|c|}
\hline \multicolumn{1}{|c|}{ Método de estimación } & Valor \\
\hline -Valor en Riesgo VaR & -0.0305634173 \\
\hline -Valor en riesgo condicional (CVaR) Expected Shortfall (ES) & -0.0348488239 \\
\hline -VaR - EVT/Distribución Gumbel & -0.0200639933 \\
\hline -VaR - EVT/Distribución generalizada del error & -0.0198973944 \\
\hline -Quasi-verosimilitud & -0.01943537 \\
\hline
\end{tabular}

Fuente: Elaboración propia

\subsubsection{Rendimientos de la acción Ecopetrol}

Como se puede observar en la figura 10, no existe tipo de comportamiento alguno relacionado con los rendimientos 
Figura 10. Linea Rendimientos de la acción de Ecopetrol

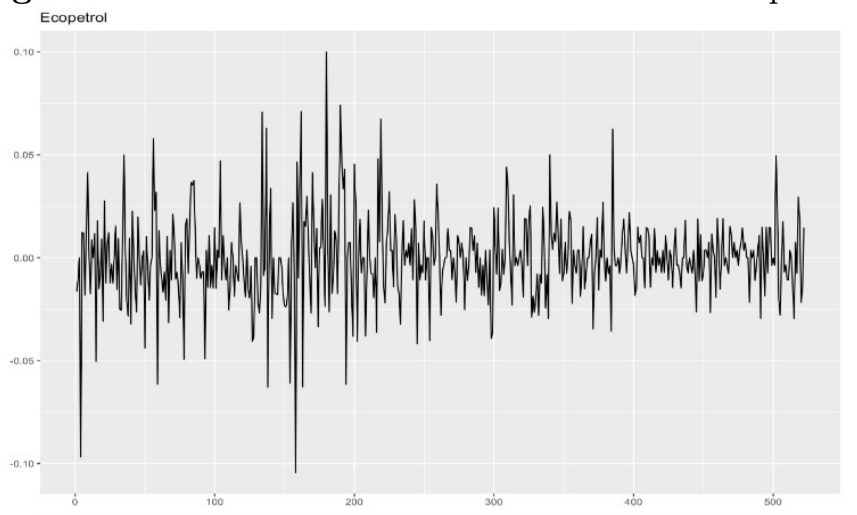

Fuente: Elaboración propia

Una vez establecido que la serie no presenta ningún tipo de tendencia, se realiza la prueba de Phillips-Perron con el fin de identificar la presencia de raíz unitaria en la serie para luego continuar con el modelado, como se puede ver a continuación:

Tabla 9. Prueba raíz unitaria acción Ecopetrol

Null Hypothesis: ECOPE has a unit root

Exogenous: Constant

Bandwidth: 1 (Newey-West automatic) using Bartlett Kernel

\begin{tabular}{|l|c|c|c|}
\hline \multirow{2}{*}{ Phillips-Perron test statistic } & Adj. t-Stat & Prob * \\
\cline { 3 - 3 } & & -20.00800 & 0.000 \\
\hline \multirow{3}{*}{ Test critical values } & $1 \%$ level & -3.442673 & \\
\cline { 2 - 3 } & $5 \%$ level & -2.866868 & \\
\cline { 2 - 3 } & $10 \%$ level & -2.569669 & \\
\hline
\end{tabular}

*Mackinnon (1996) one-sided p-values

Fuente: Elaboración propia

De acuerdo con la tabla 9, se puede observar que se rechaza la hipótesis nula de ausencia de raíz unitaria en la serie por lo que esta es estacionaria; debido a esto se procede a la selección del mejor modelo en series de tiempo para los rendimientos de "Ecopetrol" con el uso del siguiente correlograma:

Figura 11. Diagrama de autocorrelación y autocorrelación parcial de la acción de Ecopetrol

\begin{tabular}{|c|c|c|c|c|c|c|}
\hline Autocorrelation & Partial Correlation & & $\mathrm{AC}$ & PAC & Q-Stat & Prob \\
\hline I 1 & 1 & 1 & -0.052 & -0.052 & 1.4297 & 0.232 \\
\hline di & di & 2 & -0.066 & -0.069 & 3.7021 & 0.157 \\
\hline 10 & 101 & 3 & 0.068 & 0.061 & 6.1139 & 0.106 \\
\hline 1 & 1 & 4 & -0.043 & -0.041 & 7.0975 & 0.131 \\
\hline $1 \mid 1$ & $1]$ & 5 & 0.015 & 0.019 & 7.2111 & 0.205 \\
\hline
\end{tabular}

Fuente: Elaboración propia

Para el caso de los rendimientos, se encontró que el modelo $A R M A(2,2)$ con un AIC de -4.872307 es el que mejor explica el comportamiento de los rendimientos de la acción Ecopetrol. Continuando con el desarrollo del modelo como un primer acercamiento a la distribución de los rendimientos, se realiza, la prueba de Jarque-Bera para ver si existe normalidad en los residuales, como se muestra a continuación: v

Como se puede observar en la figura 12 , que con una significancia previamente establecida del $10 \%$ se rechaza la hipótesis nula de normalidad en los residuales. 
Como última fase en el modelado básico de la serie de tiempo, se realiza la prueba de heterocedasticidad, con el fin de validar el supuesto de volatilidad en los rendimientos de la serie como se muestra a continuación

Tabla 10. Prueba de heterocedasticidad ARCH para los residuales de la acción Ecopetrol

Heteroskedasticity: Test: ARCH

\begin{tabular}{|l|l|l|}
\hline F-statistic & 5.601329 & Prob. F (1,519) 0.0183 \\
\hline Obs*R- squared & 5.562877 & Prob. Chi-Square (1) 0.0183 \\
\hline \multicolumn{3}{|c|}{ Fuente: Elaboración propia }
\end{tabular}

De acuerdo con la tabla 10, se rechaza la hipótesis nula de homocedasticidad en los residuales por lo que existe la presencia de volatilidad. Siguiendo con el análisis, y considerando un nivel de significancia del $10 \%$, se puede concluir que, las estimaciones del coeficiente del modelo de corto plazo del proceso en el modelo completo son significativas.

A continuación, se presenta el resultado de la prueba de Ljung-Box para los residuales del modelo identificado:

Tabla 11. Prueba de ljung-box sobre los residuales del modelo para la acción Ecopetrol

\begin{tabular}{|c|c|c|}
\hline Q(lag) & Estadístico & p-value \\
\hline 1 & 0.002182 & 0.9627 \\
\hline 8 & 1.185344 & 1.0000 \\
\hline 14 & 3.282668 & 0.9940 \\
\hline
\end{tabular}

Fuente: Elaboración propia

Los resultados presentados en la tabla 11 permiten concluir que no hay correlación serial en los residuales del modelo identificado. Lo anterior indica que, el modelo es adecuado para capturar la correlación serial a corto plazo presente en la serie.

Con el fin de expresar el comportamiento volátil de la serie de rendimientos, se calcula el modelo GARCH teniendo en cuenta el siguiente correlograma de los residuales al cuadrado:

Figura 13. Diagrama de autocorrelación y autocorrelación parcial de los residuales al cuadrado del modelo para la acción Ecopetrol
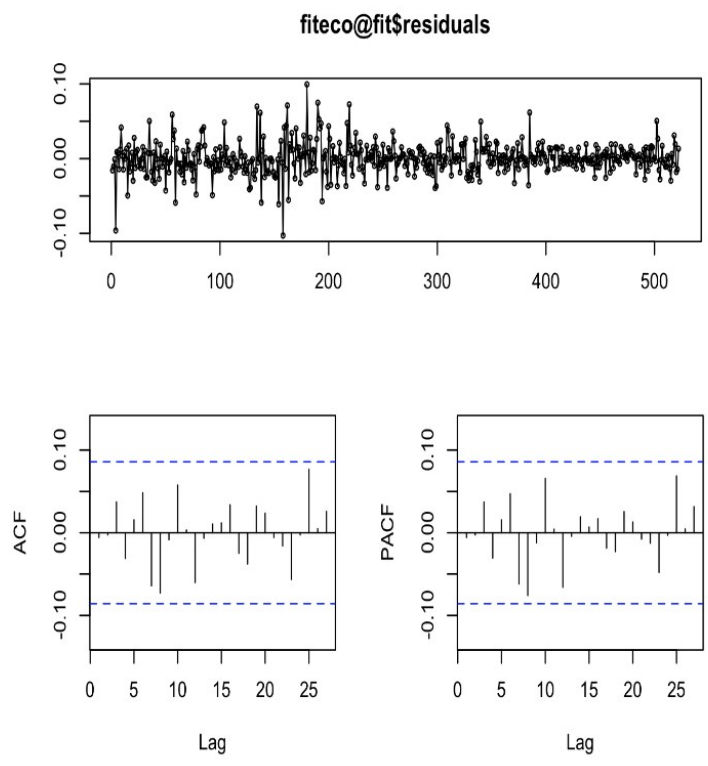

Fuente: Elaboración propia 
De acuerdo con la figura 13, el modelo que mejor captura la volatilidad de los rendimientos lo constituye un $\operatorname{GARCH}(1,1)$ con un AIC de -5.098061.

Teniendo en cuenta lo descrito anteriormente, se procede a calcular las siguientes estimaciones para el valor en riesgo:

Tabla 12. Estimaciones del VaR para la acción Ecopetrol

\begin{tabular}{|l|c|}
\hline \multicolumn{1}{|c|}{ Método de estimación } & Valor \\
\hline -Valor en Riesgo VaR & -0.0491560011 \\
\hline -Valor en riesgo condicional (CVaR) Expected Shortfall (ES) & -0.0568813340 \\
\hline -VaR - EVT/Distribución Gumbel & -0.0322694831 \\
\hline -VaR - EVT/Distribución generalizada del error & -0.0328345307 \\
\hline -Quasi-verosimilitud _ Fuente: Elaboración propia & -0.02702382 \\
\hline
\end{tabular}

Fuente: Elaboración propia

\subsubsection{Rendimientos de la acción ordinaria Bancolombia}

Se puede observar el posible comportamiento estacionario de los rendimientos de la acción Bancolombia en la siguiente figura:

Figura 14. Rendimientos de la acción ordinaria de Bancolombia

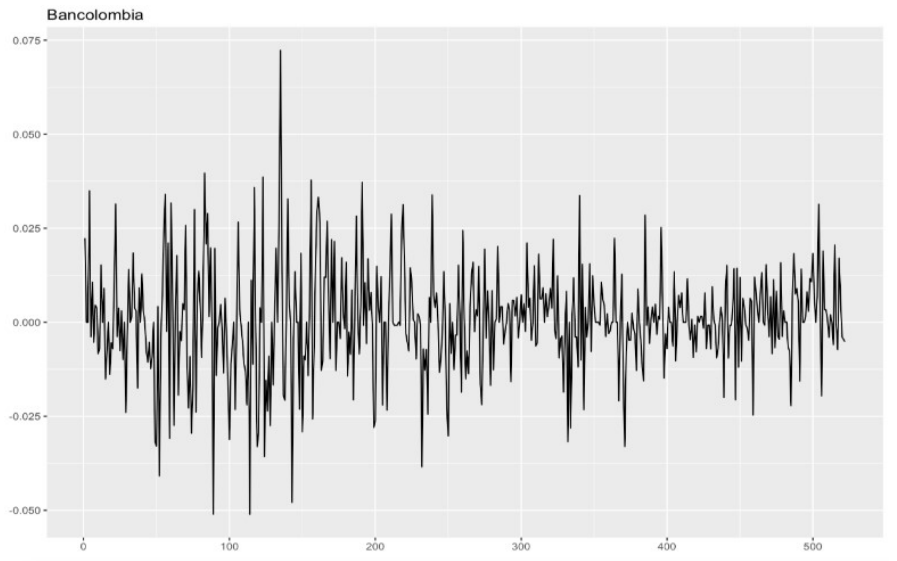

Fuente: Elaboración propia

Se puede observar en la figura 14, la falta de tendencia y un posible comportamiento estacionario de los rendimientos, por lo que se procede a verificar dicho comportamiento por medio de la prueba Phillips-Perron, como se muestra a continuación:

Tabla 13. Prueba raíz unitaria acción ordinaria Bancolombia Null Hypothesis: BCOL has a unit root

Exogenous: Constant

Bandwidth: 7 (Newey-West automatic) using Bartlett Kernel

\begin{tabular}{|l|c|c|c|}
\hline \multirow{2}{*}{ Phillips-Perron test statistic } & Adj. t-Stat & Prob * \\
\cline { 3 - 3 } & & -21.79115 & 0.000 \\
\hline \multirow{3}{*}{ Test critical values } & $1 \%$ level & -3.442673 & \\
\cline { 2 - 3 } & $5 \%$ level & -2.866868 & \\
\cline { 2 - 3 } & $10 \%$ level & -2.569669 & \\
\hline
\end{tabular}

*Mackinnon (1996) one-sided p-values

Fuente: Elaboración propia

Como se puede observar en la tabla 13, se rechaza la hipótesis nula de ausencia de raíz unitaria en la serie; por lo tanto, se deduce que es estacionaria. Debido a esta situación, se procede a la selección del mejor modelo en series de tiempo para los rendimientos "Bancolombia" con el uso del siguiente correlograma: 
Figura 15. Diagrama de autocorrelación y autocorrelación parcial de la acción ordinaria Bancolombia

\begin{tabular}{|c|c|c|c|c|c|c|}
\hline Autocorrelation & Partial Correlation & & $\mathrm{AC}$ & PAC & Q-Btat & Prob \\
\hline ון ו & ון ו & 1 & 0.048 & 0.048 & 1.1964 & 0.274 \\
\hline 1 1 & 1,1 & 2 & 0.024 & 0.022 & 1.4966 & 0.473 \\
\hline 10 & 10 & 3 & -0.040 & -0.042 & 2.3492 & 0.503 \\
\hline ומןו & ומן & 4 & 0.028 & 0.031 & 2.7605 & 0.599 \\
\hline ip & $1 p$ & 5 & 0.081 & 0.081 & 6.2343 & 0.284 \\
\hline
\end{tabular}

Fuente: Elaboración propia

Para el caso de los rendimientos se encontró que, el modelo $A R M A(2,2)$ con un AIC de -5.656407 es el que mejor explica el comportamiento de los rendimientos sin tener en cuenta el primer rezago. Siguiendo con la modelación, se realiza un primer acercamiento a la distribución de los rendimientos, a través de la prueba de Jarque-Bera, para identificar si existe o no normalidad en los residuales como se muestra a continuación:

Figura 16. Prueba de normalidad para el modelo de la acción ordinaria Bancolombia

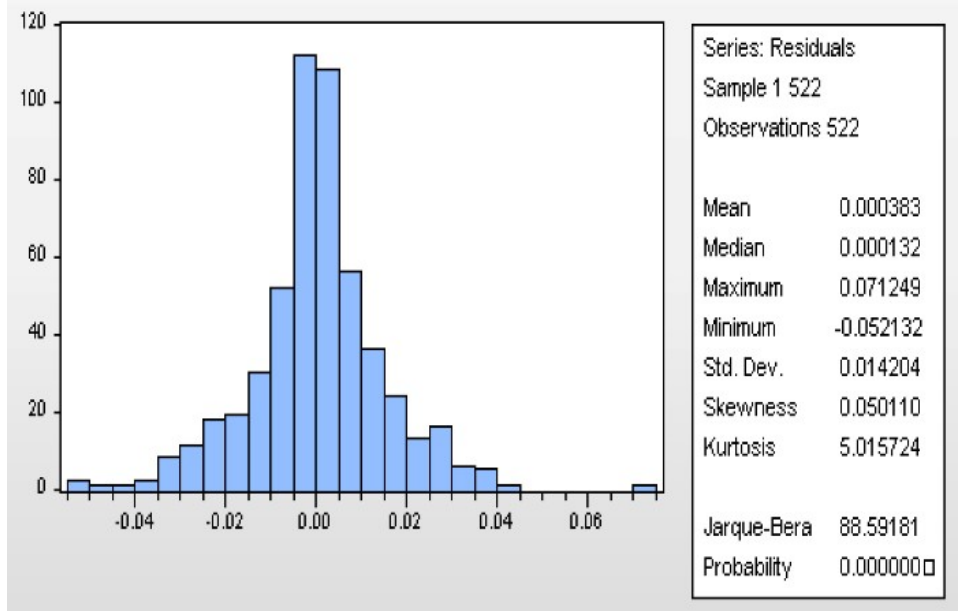

Fuente: Elaboración propia

De acuerdo con la figura 16 se rechaza la hipótesis nula de normalidad sobre los residuales en el modelo propuesto. Posteriormente a la identificación de la no normalidad en los residuales se realiza la prueba de White de Heterocedasticidad en la cual se evidencia volatilidad en los rendimientos como se muestra a continuación:

Tabla 14. Prueba de heterocedasticidad ARCH para los residuales de la acción ordinaria Bancolombia

Heteroskedasticity: Test: White

\begin{tabular}{|l|l|l|}
\hline F-statistic & 226.7127 & Prob. F (10,511) 0.0000 \\
\hline Obs*R-squared & 425.9850 & Prob. Chi-Square (10) 0.0000 \\
\hline Scaled explained SS & 842.7783 & Prob. Chi-Square (10) 0.0000 \\
\hline
\end{tabular}

Fuente: Elaboración propia

Siguiendo con el modelo, y considerando un nivel de significancia del $10 \%$, se puede concluir que las estimaciones del coeficiente del modelo de corto plazo del proceso en el modelo completo son significativas. A continuación, se presenta el resultado de la prueba de Ljung-Box para los residuales del modelo identificado. 
REMEF (The Mexican Journal of Economics and Finance) Análisis comparativo de las metodologías de estimación semiparamétricas

Tabla 15. Prueba de Ljung-box sobre los residuales del modelo para la acción ordinaria de Bancolombia

\begin{tabular}{|c|c|c|}
\hline Q(lag) & Estadístico & p-value \\
\hline 1 & 0.18437 & 0.6676 \\
\hline 10 & 4.085 & 0.9434 \\
\hline 15 & 10.01 & 0.8191 \\
\hline \multicolumn{2}{|c|}{ Fuente: Elaboración propia } \\
\hline
\end{tabular}

Fuente: Elaboración propia

Los resultados presentados en la tabla anterior permiten concluir que no hay correlación serial en los residuales del modelo identificado. Lo anterior indica que el modelo es adecuado para capturar la correlación serial a corto plazo presente en la serie.

Con el fin de expresar el comportamiento volátil de la serie de rendimientos, se calcula el modelo GARCH teniendo en cuenta, el siguiente correlograma de los residuales al cuadrado:

Figura 17. Diagrama de autocorrelación y autocorrelación parcial de los residuales al cuadrado del modelo para la acción ordinaria de Bancolombia
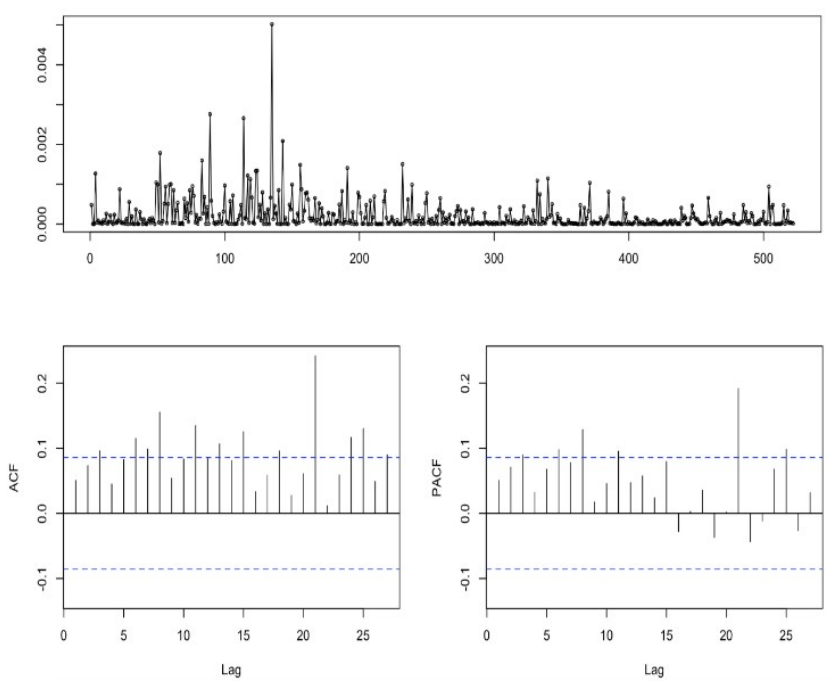

Fuente: Elaboración propia

Se estableció de acuerdo con la figura 17, que el modelo, que mejor captura la volatilidad de los rendimientos, lo constituye un $G A R C H(1,1)$ con un AIC de -5.826302 .

Teniendo en cuenta lo descrito anteriormente, se calcularon las siguientes estimaciones para el valor en riesgo:

Tabla 16. Estimaciones del VaR para la acción ordinaria Bancolombia

\begin{tabular}{|l|c|}
\hline \multicolumn{1}{|c|}{ Método de estimación } & Valor \\
\hline -Valor en Riesgo VaR & -0.0332057961 \\
\hline -Valor en riesgo condicional (CVaR) Expected Shortfall (ES) & -0.0376404786 \\
\hline -VaR - EVT/Distribución Gumbel & -0.0217986380 \\
\hline -VaR - EVT/Distribución generalizada del error & -0.0213964142 \\
\hline -Quasi-verosimilitud & -0.018491 \\
\hline
\end{tabular}

Fuente: Elaboración propia

\subsection{Estimación del VaR por medio de modelos VineCópula}

En ninguno de los casos mencionados anteriormente se tuvo en cuenta la estructura de dependencia no lineal entre los activos para la estimación del VaR. Para considerar metodología es necesario tener en cuenta la relación entre los activos dentro de un mismo 
portafolio de inversión; en este caso se construyó un portafolio constituido con los rendimientos de los siguientes activos:

- Preferencial Bancolombia (P)

- Grupo Sura (G)

- Ecopetrol (E)

Teniendo en cuenta los anteriores análisis y la descripción de las 522 observaciones para cada uno de los activos desde el 10 de junio de 2015 hasta el 10 de junio de 2017; se obtuvieron los siguientes modelos previamente calculados con los que se desarrolla esta metodología:

$$
\begin{gathered}
P_{t}=6,8493 e^{-04}+0,3753 P_{t-1}+0,2872 P_{t-2}-0,4249 Z_{t-1}-0,1925 Z_{t-2}+Z_{t} \\
G_{t}=1,8139 e^{-04}+0,9475 G_{t-1}-8,020476 e^{-03} G_{t-2}-Z_{t} \\
E_{t}=-7,3202^{-04}-0,3829 E_{t-1}-0,08098 E_{t-2}+0,3382 Z_{t-1}+Z_{t}
\end{gathered}
$$

En cuanto a explicar la volatilidad de los rendimientos de los activos financieros en el portafolio, los coeficientes de los modelos GARCH calculados se muestran a continuación:

Tabla 17. Estimación parámetros modelos GARCH

\begin{tabular}{|c|c|c|c|}
\hline Parámetro & $\begin{array}{c}\text { Preferencial } \\
\text { Bancolombia }\end{array}$ & Grupo Sura & Ecopetrol \\
\hline$\omega$ & $3.7387 \mathrm{e}-06$ & $7.198519 \mathrm{e}-06$ & $5.1556 \mathrm{e}-06$ \\
\hline$\alpha_{1}$ & $5.7285 \mathrm{e}-02$ & $9.434554 \mathrm{e}-02$ & $5.5947 \mathrm{e}-02$ \\
\hline$\beta_{1}$ & 0.9241 & 0.8716184 & 0.9367 \\
\hline shape & 6.054365 & 3.883865 & 4.034393 \\
\hline \multicolumn{4}{|c}{ Fuente: Elaboración propia } \\
\hline
\end{tabular}

Una vez realizadas las estimaciones, se verificó la distribución de los residuales; en este caso, como en las modelaciones anteriores, los residuales de ninguno de los rendimientos presenta distribución normal, por lo que se evidencia un comportamiento leptocúrtico de los datos y las colas pesadas en estos. Se hicieron supuestos sobre una posible distribución t presente en los residuales de cada uno de los modelos ajustados.

A continuación, se muestran los diagramas qplot que permiten identificar estos supuestos:

Figura 18. Diagramas Qplot para las acciones Preferencial Bancolombia, Grupo Sura y Ecopetrol
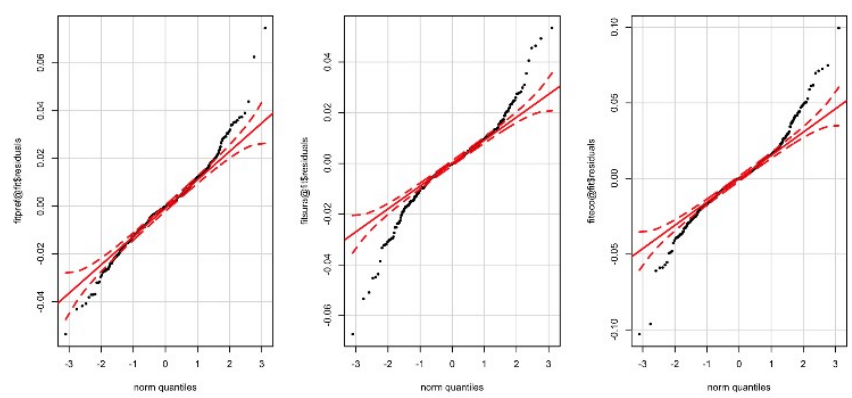

Fuente: Elaboración propia

Teniendo en cuenta el supuesto de que los residuales del modelo presentan una distribución t por las características en los diagramas presentados, se procede a calcular los 
grados de libertad respectivos para estas distribuciones por medio de máxima verosimilitud a través del algoritmo de Newton-Rapson para maximización por medio del paquete (maxLik) del lenguaje R.

En la siguiente tabla se pueden observar las estimaciones para cada uno de los grados de libertad:

Tabla 18. Estimación de los grados de libertad para la distribución de los residuales de los modelos GARCH calculados

\begin{tabular}{|l|c|c|}
\hline \multicolumn{1}{|c|}{ Residuales } & Estimación & $\mathbf{P r}(>\mathbf{t})$ \\
\hline Preferencial Bancolombia & 57.1291309 & $<2 \mathrm{e}-16^{* * *}$ \\
\hline Grupo Sura & 23.2779885 & $<2 \mathrm{e}-16^{* * *}$ \\
\hline Ecopetrol & 14.37335 & $<2 \mathrm{e}-16^{* * *}$ \\
\hline
\end{tabular}

Fuente: Elaboración propia

De acuerdo con la tabla 18, se puede observar que la estimación por cada una de las series resulta ser significativa; por lo tanto, la maximización vía Newton-Rapson estima de manera correcta los grados de libertad de los residuales de los modelos calculados.

\subsubsection{Selección de la Cópula}

Conociendo las distribuciones marginales de los residuales de los rendimientos, se transformaron en datos cópula reescribiéndolos en términos de probabilidades en orden para seleccionar la cópula que logre capturar la dependencia entre los datos, los cuales están denotados como $u_{P}$ para "preferencial Bancolombia, $u_{S}$ para "Grupo Sura" y $u_{E}$ para "Ecopetrol. Por medio de la función RVineStructureSelect del paquete VineCópula se conoce la forma específica del árbol D-vine construido con la siguiente estructura:

Figura 19. Estructura del árbol D-Vine Portafolio

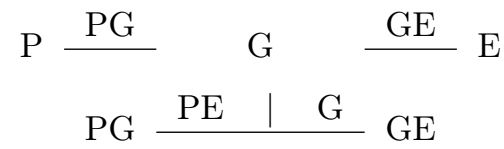

Fuente: Elaboración propia

Figura 20. Matriz de Relación para cópulas

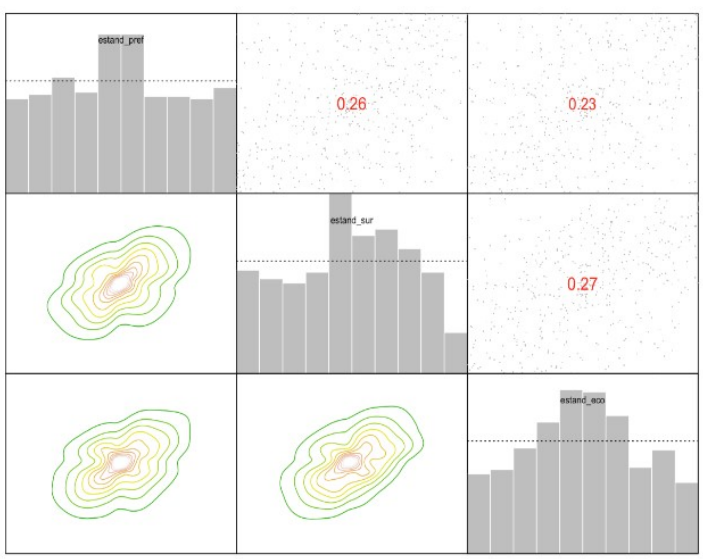

Fuente: Elaboración propia

En la figura 20 se puede observar la relación de dependencia no lineal entre los rendimientos de los activos financieros por medio del Tau de Kendall, así como la forma de las cópulas asociadas a la dependencia entre las series. Siguiendo a (Czado, 2013) la 
estructura PCC para el conjunto de rendimientos se escribe como:

$$
C\left(u_{P}, u_{S}, u_{E}\right)=C_{P S} \cdot C_{S E} \cdot C_{P E \mid S}{ }^{6}
$$

donde la estructura D-vine está construida a partir de los siguientes pares de cópulas:

- Árbol 1:

$$
\begin{gathered}
C\left(u_{P}, u_{S}\right)=-\frac{1}{\theta_{1}} \log \left[1+\frac{\left(\exp \left(-\theta_{1} u_{P}\right)-1\right)\left(\exp \left(-\theta_{1} u_{S}\right)-1\right)}{\left(\exp \left(-\theta_{1}\right)-1\right)}\right] \\
\left(u_{S}, u_{E}\right)=-\frac{1}{\theta_{2}} \log \left[1+\frac{\left(\exp \left(-\theta_{2} u_{S}\right)-1\right)\left(\exp \left(-\theta_{2} u_{E}\right)-1\right)}{\left(\exp \left(-\theta_{2}\right)-1\right)}\right]
\end{gathered}
$$

- Árbol 2:

$$
C\left(u_{P}, u_{E} \mid u_{S}\right)=\frac{1}{2 \pi \sqrt{1-\rho^{2}}}\left(1+\frac{u_{P S}^{2}-2 u_{P S} u_{S E}+u_{S E}^{2}}{v\left(1-\rho^{2}\right)}\right)^{-\frac{v+2}{2}}
$$

Una vez identificada la estructura D-Vine se procede a la estimación de los parámetros establecidos por cada cópula en cada uno de los árboles $\theta_{1}, \theta_{2}, v, \rho$ por medio de máxima verosimilitud con la función RVineStructureSelect, la cual arroja los valores estimados de los parámetros y una matriz especifica identificando la familia de la cópula asociada al determinado árbol. Posteriormente se genera por medio de la función RVineSim (10000) triplas de valores que se convierten posteriormente en los cuantiles de las distribuciones t previamente estimadas.

Finalmente, las posibles pérdidas se ponderan con las predicciones de la parte volátil del modelo $\left(\sigma_{i}, t\right)$ en el momento $t+1$, calculando la parte en media móvil $Z_{t}$ de los modelos $X_{1, t}, X_{2, t} y X_{3, t}$ obteniendo así una distribución de posibles errores sobre los que se realiza el cálculo de VaR correspondiente.

Teniendo en cuenta la siguiente estructura del modelo calculado con el paquete $\mathrm{R}$ Rugarch para evaluar la precisión de las estimaciones del VaR, se realizó la estimación al $90 \%, 95 \%$ y $99 \%$ de nivel de confianza mediante el siguiente procedimiento:

$$
\begin{gathered}
r_{i, t}=\mu+\phi_{1}\left(r_{i, t-1}-\mu\right)+\ldots+\theta_{1} \varepsilon_{t-1}+\ldots+\varepsilon_{i, t} \\
\varepsilon_{i, t}=\sigma_{i, t} \cdot \eta_{i, t} \\
\sigma_{i, t}^{2}=\omega_{i}+\alpha_{i} \epsilon_{i, t-1}^{2}+\beta_{i} \sigma_{i, t-1}^{2}
\end{gathered}
$$

donde $\eta_{i, t}$ es el vector simulado de los tres activos

Calculando las expresiones previas de la distribución de la cartera después de hacer todas las predicciones, se procede a calcular el valor en riesgo al $90 \%, 95 \%$ y $99 \%$ de nivel de confianza con pesos iguales para los retornos $w_{i}=\frac{1}{3}$. A continuación, se muestra el cálculo del valor en riesgo

Tabla 19. Estimación del VaR para el portafolio

\begin{tabular}{|c|c|c|}
\hline $90 \%$ & $95 \%$ & $99 \%$ \\
\hline 0.03293495 & 0.04277162 & 0.06326884 \\
\hline \multicolumn{2}{|c|}{ Fuente: Elaboración propia }
\end{tabular}

\footnotetext{
${ }^{6}$ Preferencial Bancolombia (P), Grupo Sura (S), Ecopetrol (E)
} 
REMEF (The Mexican Journal of Economics and Finance) Análisis comparativo de las metodologías de estimación semiparamétricas

En cuanto al desempeño establecido por parte de las metodologías, el uso de distribuciones de valores extremos es comúnmente mencionado varios los analistas como mejor medida para retener las colas pesadas de las distribuciones de los retornos para los activos financieros. Adicionalmente, el valor en risgo condicional resulta ser una de las alternativas preferidas al tener en cuenta la forma completa de la cola para la perdida esperada, en vez de un único punto.

Para los modelos de volatilidad usando la estimación proveniente de la quasi-verosimilitud se uso de backtesting, se obtuvieron los siguientes resultados usando la prueba incondicional de Kupiec y condicional de Christoffersen:

Tabla 20. Backtesting para estimacion GARCH quasi-verosimil

\begin{tabular}{|c|c|c|c|}
\hline $\begin{array}{c}\text { Modelo } \\
\text { implementado }\end{array}$ & $\begin{array}{c}\text { Excedencias } \\
\text { actuales/esperadas }\end{array}$ & $\begin{array}{c}\text { P-valores para test de } \\
\text { Kupiec/Christoffersen }\end{array}$ & $\begin{array}{c}\text { Decisión (El modelo } \\
\text { se considera:) }\end{array}$ \\
\hline Pref. Ban & $10 / 5$ & $0.06188 / 0.07031$ & Exacto \\
\hline G. Sura & $14 / 5$ & $0.00139 / 0.00412$ & Inexacto \\
\hline Ecopetrol & $11 / 5$ & $0.02680 / 0.06794$ & Indecisión \\
\hline Bancolombia & $11 / 5$ & $0.02680 / 0.04073$ & Inexacto \\
\hline \multicolumn{2}{|r}{ Fuente: Elaboración propia }
\end{tabular}

De este backtesting se pudo concluir que en todos los casos se presentó mayor numero de excedencias a las esperadas por el modelo. En el caso del uso de Vine Copulas un cálculo para backtesting representa un alto costo computacional y dispendioso en implementación por lo cual no fue implementado sino a manera de resultado para la metodología propuesta. Esta ultima metodología se estimó para todo el portafolio en conjunto.

\section{Conclusiones}

De acuerdo con la investigación realizada se puede concluir que la estimación para el valor en riesgo por medio de los modelos vinecópula logran describir de manera adecuada el comportamiento de la volatilidad incesante de los activos relacionados (acción preferencial Bancolombia, Grupo Sura y Ecopetrol) así como la estructura de dependencia no lineal capturada por otras metodologías

De otro lado el método por quasi-verosimilitud da un primer acercamiento libre de supuestos sobre la distribución de los residuales del modelo GARCH calculado para la estimación del valor en riesgo

Las metodologías semiparamétricas como el EVT logran capturar el comportamiento en colas pesadas de las distribuciones de los datos, permitiendo así una estimación mejor a la estadística tradicional en la estimación del valor en riesgo. Aunque hay otras metodologías que permiten la optimización de portafolios con activos cuando los rendimientos se modelan mediante procesos log-estables como la presentada en (Climent-Hernández, 2017), que permite mejorar la eficiencia frente a los modelos log-gaussianos, los modelos Vine-Cópula permiten abrir una nueva ventana de investigación en las que se pueden establecer comparaciones de rendimiento y backtesting entre estas dos metodologías.

Finalmente, de acuerdo con (Acerbi Tasche, 2002) que manifiesta que el VaR no es apto para describir los riesgos de una cartera y que existen alternativas más prácticas en la clase de medidas de riesgo como el CVaR, cuyo objetivo es centrarse en los activos menos rentables, este artículo presenta una metodología para la estimación del riesgo en portafolios que involucran cópulas y que permite acercarse de manera más acertada a la realidad del mercado bursátil colombiano. En trabajos futuros se desea extender la metodología de cópulas y el acercamiento semiparametrico para el cálculo otro indicador de riesgo financiero, sin renunciar a las ventajas del VaR. 


\section{Referencias}

Acerbi, C., Tasche, D. (2002). Expected shortfall: a natural coherent alternative to value at risk. Economic notes, 31(2), 379-388.

Alzghool, R. (2017, 04). Parameters estimation for GARCH (p,q) model: QL and AQL approaches. Electronic Journal of Applied Statistical Analysis EJASA, 3-16.

Brechmann, E., Schepsmeier, U. (2013). Modeling Dependence with C- and D-Vine Copulas: The R Package CDVine. Journal of Statistical Software, 52(3), 1-27.

Breuer, T. (2008). VaR, CVaR, and EVT. Risk Training.

Caicedo, S., Enrique, R. (2014). Valor en riesgo del portafolio de TES de los bancos colombianos. Bogotá: Doctoral Dissertation, Universidad del Rosario. Retrieved from repository.urosario. edu.co/bitstream/handle/10336/8909/1136881062-2014.pdf? .

Cardozo, P. (2004). Valor en riesgo de los activos financieros colombianos aplicando la teoria de valor extremo. Borradores de economía, 1-39. Retrieved from http://www.banrep.gov.co/sites/default/ files/publicaciones/pdfs/borra304.pdf

Climent-Hernández, J. A. (2017). Portafolios de dispersión mínima con rendimientos log-estables. Revista mexicana de economía y finanzas, 12(2), 49-69.

Czado, C. Aleksey. M. (2010, 10 1). Bayesian inference for multivariate copulas using pair-copula constructions. Journal of Financial Econometrics, 8(4), 546.

Czado, C. (2013). Vine copulas and their applications to financial data . AFMathConf 2013.

Embrechts, P Hoing, A. (2006). Extreme VaR scenarios in higher dimensions.

Embrechts, P. (2016). VaR-based Risk Managment sense and (Non-) Sensibility. RiskLab Department of Mathematics ETH Zurich.

Fan J Gu J. (2003). Semiparametric estimation of Value Risk. Econometrics Journal, 261-290. Retrieved from https://pdfs.semanticscholar.org/fdad/86e5414f67327e41e0b0c7cecf30c87b8406.pdf

Feria, J Oliver, M. (2006). Valor en Riesgo (VeR): conceptos, parámetros y utilidad. Universia Business Review, 10, 66-79.

Fernandez, V. (2003). Extreme Value Theory: Value at risk and returns dependence around the world.

Gu, J. F. (2003). Semiparametric Estimation of Value-at-Risk. Department of Statistics.

Hao Li, Xiao Fan, Yu Li, Yue Zhou, Ze Jin Zhao Liu. (2014). Aproaches to VaR. Stanford University, 44.

Hofmann, M Czado, C. (2011). Assessing the VaR of a portfolio using D-vine copula based multivariate GARCH models. Lehrstuhl fur Mathematische Statistik, 1-33.

Joe, H. (2015). Dependence Modeling with Copulas.

Larsen, J; Solli M Ingebrigt P. (2013). Risk Modelling using Vine Copulas. Norwegian University of Science and Technology.

Lei QI Jianqing FAN . (2014, May 20). Quasi-Maximum Likelihood Estimation of GARCH Models With Heavy-Tailed Likelihoods.

Lei, Fan J Xiu D. (2014). Quasi-Maximum Likelihood Estimation of GARCH models with heavy-tailed likelihoods. University of Chicago. Retrieved from http://dachxiu.chicagobooth.edu/download/ NGQMLE.pdf

Mariño, D Melo, L. (2016). Regresión Cuantílica Dinámica para la Medición del Valor en Riesgo: una Aplicación a Datos Colombianos. Borradores de Economía, 1-25.

Melo V, Luis Becerra C, Oscar. (2005). Medidas de riesgo, caracteristicas y tecnicas de medicion: una Aplicacion del VaR y el ES a la tasa interbancaria de Colombia. Borradores de Economía, 1-75.

Nelsen, R. B. (2006). An introduction to copulas. Springer Series in Statistics.

Nicole Krämer Ulf Schepsmeier. (2011). Introduction to vine copulas. NIPS Workshop.

RISK. (2014, 12). Expected Shortfall: The future? RISK.

Supefinanciera. (2007). Retrieved from https://www.superfinanciera.gov.co/inicio/normativa/normativa-general/ circular-basica-contable-y-financiera-circular-externa--de---15466

Superfinanciera. (2007). Retrieved from https://www.superfinanciera.gov.co/jsp/loader.jsf?1Servicio= Publicaciones\&lTipo=publicaciones\&lFuncion=loadContenidoPublicacion\&id=15466

Timotheos A, Benos A Degiannakis S. (2003). The Use of GARCH Models in VaR Estimation. macro.soc.uoc.gr, 36 .

Triana, D., Torres Aponte, L., Alba, M., Pineda-Ríos, W. (2017). Estimación Bayesiana para el cálculo del Valor en Riesgo (VaR) en modelos de series financieras con relaciones de dependencia no lineal en Colombia. Comunicaciones en Estadistica Universidad Santo Tomas ISSN: 2027-3355 ISSN Online: 2339-3076, 11(2), 171-189. 